\title{
Anomalous mirror symmetry breaking in a model insulating cuprate $\mathrm{Sr}_{2} \mathrm{CuO}_{2} \mathrm{Cl}_{2}$
}

\author{
A. de la Torre,${ }^{1,2}$ K. L. Seyler,${ }^{1,2}$ L. Zhao, ${ }^{3}$ S. Di Matteo, ${ }^{4}$ M. S. Scheurer,${ }^{5}$ \\ Y. Li, ${ }^{6}$ B. Yu, ${ }^{6}$ M. Greven, ${ }^{6}$ S. Sachdev, ${ }^{5}$ M. R. Norman, ${ }^{7}$ and D. Hsieh ${ }^{1,2}$ \\ ${ }^{1}$ Department of Physics, California Institute of Technology, Pasadena, CA 91125, USA \\ ${ }^{2}$ Institute for Quantum Information and Matter, \\ California Institute of Technology, Pasadena, CA 91125, USA \\ ${ }^{3}$ Department of Physics, University of Michigan, \\ 450 Church Street, Ann Arbor, Michigan 48109, USA \\ ${ }^{4}$ Universite de Rennes, CNRS UMR 6251 Institut de Physique de Rennes, F-35708 Rennes, France \\ ${ }^{5}$ Department of Physics, Harvard University, Cambridge, MA 02138, USA \\ ${ }^{6}$ School of Physics and Astronomy, University of Minnesota, Minneapolis, Minnesota 55455, USA \\ ${ }^{7}$ Materials Science Division, Argonne National Laboratory, Argonne, IL 60439, USA
}

(Dated: August 18, 2020)

Understanding the complex phase diagram of cuprate superconductors is an outstanding challenge [1]. The most actively studied questions surround the nature of the pseudogap and strange metal states and their relationship to superconductivity [2-4]. In contrast, there is general agreement that the low energy physics of the Mott insulating parent state is well captured by a two-dimensional spin $S=1 / 2$ antiferromagnetic (AFM) Heisenberg model [5]. However, recent observations of a large thermal Hall conductivity in several parent cuprates appear to defy this simple model and suggest proximity to a magnetochiral state that breaks all mirror planes perpendicular to the $\mathrm{CuO}_{2}$ layers $[6-10]$. Here we use optical second harmonic generation to directly resolve the point group symmetries of the model parent cuprate $\mathrm{Sr}_{2} \mathrm{CuO}_{2} \mathrm{Cl}_{2}$. We report evidence of an order parameter $\Phi$ that breaks all perpendicular mirror planes and is consistent with a magneto-chiral state in zero magnetic field. Although $\Phi$ is clearly coupled to the AFM order parameter, we are unable to realize its timereversed partner $(-\Phi)$ by thermal cycling through the AFM transition temperature $\left(T_{\mathrm{N}} \approx 260 \mathrm{~K}\right)$ or by sampling different spatial locations. This suggests that $\Phi$ onsets above $T_{\mathrm{N}}$ and may be relevant to the mechanism of pseudogap formation.

The single layer oxychloride cuprate $\mathrm{Sr}_{2} \mathrm{CuO}_{2} \mathrm{Cl}_{2}$ is an ideal parent material for studying subtle magnetic symmetry breaking effects because $T_{\mathrm{N}}$ is easily accessible and because its crystallographic structure (space group 139, I4/ $\mathrm{mmm}$ ) has exceptionally high symmetry. As shown in Fig. 1(a), $\mathrm{Sr}_{2} \mathrm{CuO}_{2} \mathrm{Cl}_{2}$ consists of tetragonal $\mathrm{CuO}_{2}$ planes separated by $\mathrm{Sr}_{2} \mathrm{Cl}_{2}$ buffer layers. Unlike other commonly studied cuprates [4, no long-range tilting/rotation of the $\mathrm{CuO}_{4} \mathrm{Cl}_{2}$ octahedra or structural modulations are present either above or below $T_{\mathrm{N}}$ in $\mathrm{Sr}_{2} \mathrm{CuO}_{2} \mathrm{Cl}_{2}$ [11, 12]. This structure results in a very small magnetic anisotropy [13 16], extremely weak interlayer coupling due to frustration of the inter- layer exchange [16], and an absence of DzyaloshinskiiMoriya (DM) interactions, making $\mathrm{Sr}_{2} \mathrm{CuO}_{2} \mathrm{Cl}_{2}$ a model 2D Heisenberg antiferromagnet.

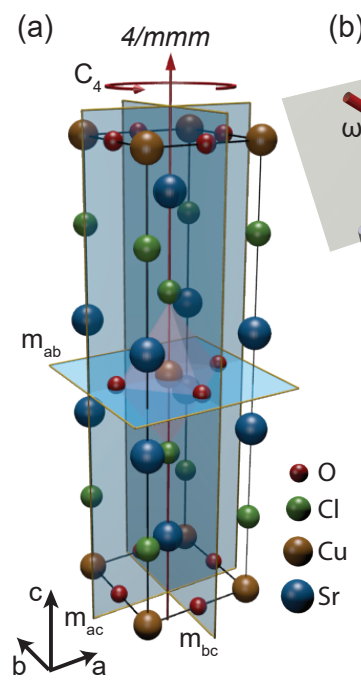

(b)

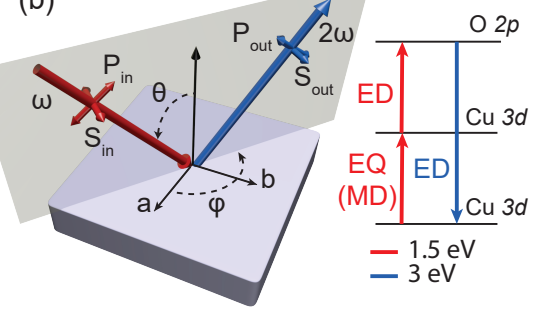

(c)

$\quad \therefore 800 \mathrm{~nm} \quad \theta=11^{\circ}$

$\triangle 800 \mathrm{~nm} \quad \theta=0^{\circ}$

$\square 1200 \mathrm{~nm} \theta=11^{\circ}$

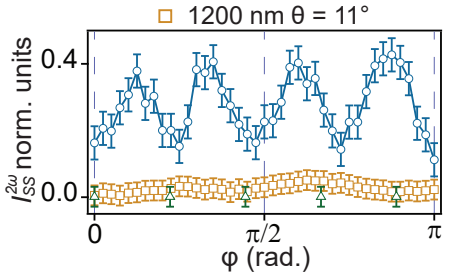

FIG. 1. (a) Unit cell of $\mathrm{Sr}_{2} \mathrm{CuO}_{2} \mathrm{Cl}_{2}$ showing the generators $\left(\mathrm{m}_{b c}, \mathrm{~m}_{a c}, \mathrm{~m}_{a b}\right.$ and $\left.\mathrm{C}_{4}\right)$ of the tetragonal $4 / \mathrm{mmm}$ point group. (b) Schematic of the RA-SHG setup. The scattering plane angle $(\varphi)$, incidence angle $(\theta)$ and input and output electric field polarization ( $\mathrm{S}$ or $\mathrm{P}$ ) are varied in our experiment. The states involved in the SHG processes discussed in the main text are shown in the inset. (c) $\varphi$-dependence $(0 \rightarrow \pi$ shown for clarity) of the SHG intensity from $\mathrm{Sr}_{2} \mathrm{CuO}_{2} \mathrm{Cl}_{2}$ at different incident angles and wavelengths measured at $T=300 \mathrm{~K}$ in the $\mathrm{S}_{\text {in }} \mathrm{S}_{\text {out }}$ (abbreviated SS) channel. Fluences and counting times were held constant. Error bars represent the statistical uncertainty. The $800 \mathrm{~nm}(\sim 1.5 \mathrm{eV}) \theta=11^{\circ}$ curve is offset vertically for clarity.

Second harmonic generation (SHG) is governed by high rank $(>2)$ nonlinear optical susceptibility tensors. Therefore it is much more sensitive to the crystallographic and magnetic point group symmetries of a crystal compared to typical linear optical responses [17. To resolve the SHG tensor structures of $\mathrm{Sr}_{2} \mathrm{CuO}_{2} \mathrm{Cl}_{2}$, we performed SHG rotational anisotropy (RA) and spec- 


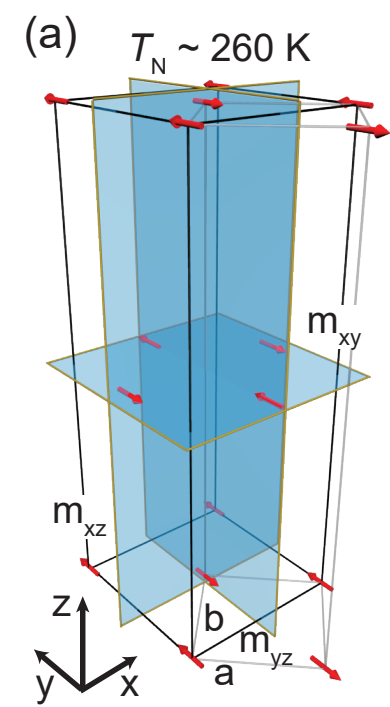

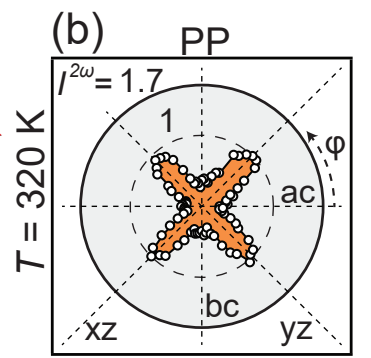
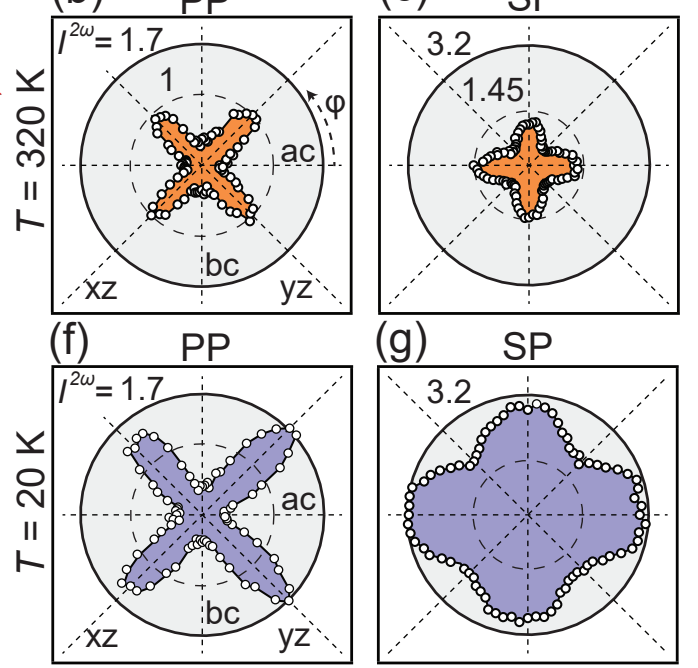

(c)

(d)

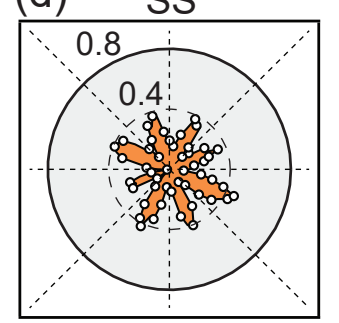

(g) SP

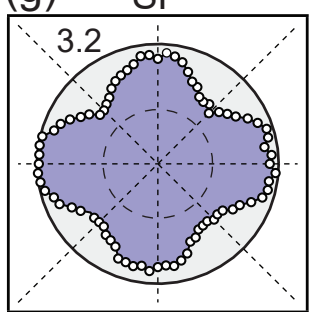

(h)

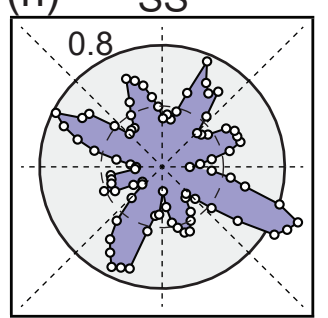

(e) PS

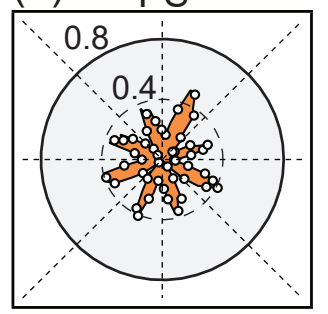

(i)

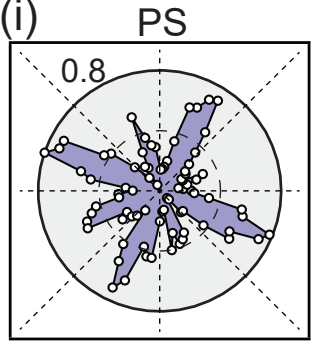

FIG. 2. (a) AFM structure of $\mathrm{Sr}_{2} \mathrm{CuO}_{2} \mathrm{Cl}_{2}$ showing the spatial symmetry generators $\left(\mathrm{m}_{y z}, \mathrm{~m}_{x z}\right.$ and $\left.\mathrm{m}_{x y}\right)$ of its orthorhombic $m m m 1^{\prime}$ point group. Moments are aligned along the $y$-axis [110] direction. Although time-reversal symmetry is locally broken at each $\mathrm{Cu}$ site, it is restored upon translation by an in-plane lattice vector. Therefore the time-reversal operator $1^{\prime}$ is a symmetry of the point group. RA data for four polarization channels measured at (b)-(e) $T=320 \mathrm{~K}$ (above $T_{\mathrm{N}}$ ) and (f)-(i) $T=20 \mathrm{~K}$. All data sets are normalized to the maximum intensity in the PP channel at $T=320 \mathrm{~K}$. There is larger statistical noise in the S-output channels due to their extremely weak intensity.

troscopy measurements in reflection mode from (001) cleaved single crystals [18. Figure 1(b) provides a depiction of our setup, showing all of the degrees of freedom used in our experiment.

We first examine the SHG response of $\mathrm{Sr}_{2} \mathrm{CuO}_{2} \mathrm{Cl}_{2}$ above $T_{\mathrm{N}}$. The $4 / \mathrm{mmm}$ point group respects inversion symmetry and so no bulk electric-dipole (ED) SHG is expected. However, surface ED SHG and higher multipole bulk processes such as electric quadrupole (EQ) SHG are allowed. These contributions are readily distinguished in the SS polarization channel [Fig. 11(b)] because ED SHG from the (001) surface is forbidden by symmetry whereas the bulk EQ SHG intensity should scale as $I_{\mathrm{SS}}^{2 \omega} \propto \sin ^{2} \theta \sin ^{2} 4 \varphi$ [19], where $\theta$ is the angle of incidence and $\varphi$ is the scattering plane angle. As shown in Fig. 1 (c), the SHG intensity from $\mathrm{Sr}_{2} \mathrm{CuO}_{2} \mathrm{Cl}_{2}(001)$ measured using $800 \mathrm{~nm}$ incident light vanishes at normal incidence $\left(\theta=0^{\circ}\right)$ but becomes finite, albeit very weak $\left(\sim 10^{4}\right.$ times weaker than GaAs [19]), at oblique incidence $\left(\theta=11^{\circ}\right)$ with a clear $\sin ^{2} 4 \varphi$ dependence. This indicates predominant sensitivity to bulk EQ SHG and consistency with a $4 / \mathrm{mmm}$ point group. Moreover, we find no detectable SHG using $1200 \mathrm{~nm}(1 \mathrm{eV})$ incident light [Fig. 1( (c)]. Given that previous optical conductivity results on $\mathrm{Sr}_{2} \mathrm{CuO}_{2} \mathrm{Cl}_{2}$ show an ED forbidden $\mathrm{Cu} d$ - $d$ excitation feature near $1.5 \mathrm{eV}(800 \mathrm{~nm})$ and a broad band of ED allowed $\mathrm{O} 2 p$ to $\mathrm{Cu} 3 d$ charge transfer excitations around 2-3 eV [20, we attribute our observed SHG signal to a two-photon process resonantly enhanced by an EQ $d-d$ transition [inset Fig. 11(b)].
A comparison of RA patterns obtained above and below $T_{\mathrm{N}}$ under all different polarization geometries is shown in Fig. 2, The $T=320 \mathrm{~K}$ data exhibit four-fold $\left(\mathrm{C}_{4}\right)$ rotational symmetry about the $c$-axis and mirror symmetry about the $a c$ and $b c$ planes as well as the diagonal $x z$ and $y z$ planes, which are all elements of the $4 / \mathrm{mmm}$ point group. These data are again consistent with bulk EQ SHG and incompatible with surface ED SHG because the latter is forbidden in the SS and PS channels and is $\varphi$-independent in the PP and SP channels [19. At $T=20 \mathrm{~K}$, the $\mathrm{SHG}$ intensity is generally larger than at $T=320 \mathrm{~K}$ in all polarization channels. However, while the PP and SP patterns retain all the symmetries of the $T=320 \mathrm{~K}$ data, the PS and SS patterns retain $\mathrm{C}_{4}$ but break the $a c, b c, x z$ and $y z$ mirror planes, manifested via a change from uniform to alternating lobe intensity.

As shown in Figure 3, the change in the RA patterns observed in the S-output channels onsets near $T_{\mathrm{N}}$ and gradually becomes more pronounced upon cooling. The absence of thermal hysteresis is consistent with a continuous phase transition at $T_{\mathrm{N}}$. Although this suggests that the change in RA patterns is correlated with AFM ordering, it cannot explain the observed symmetry lowering. Below $T_{\mathrm{N}}$, spins on the $\mathrm{Cu}$ sites of $\mathrm{Sr}_{2} \mathrm{CuO}_{2} \mathrm{Cl}_{2}$ adopt a collinear AFM arrangement characterized by an ordering wave vector $\left(\frac{1}{2}, \frac{1}{2}, 0\right)$, with moments aligned along the [110] axis [21. This spin structure is described by an orthorhombic magnetic point group $m m m 1^{\prime}$, whose generators consist of three mirror operators about the $x y$, $x z$, and $y z$ planes as well as the time-reversal operator $1^{\prime}$ 


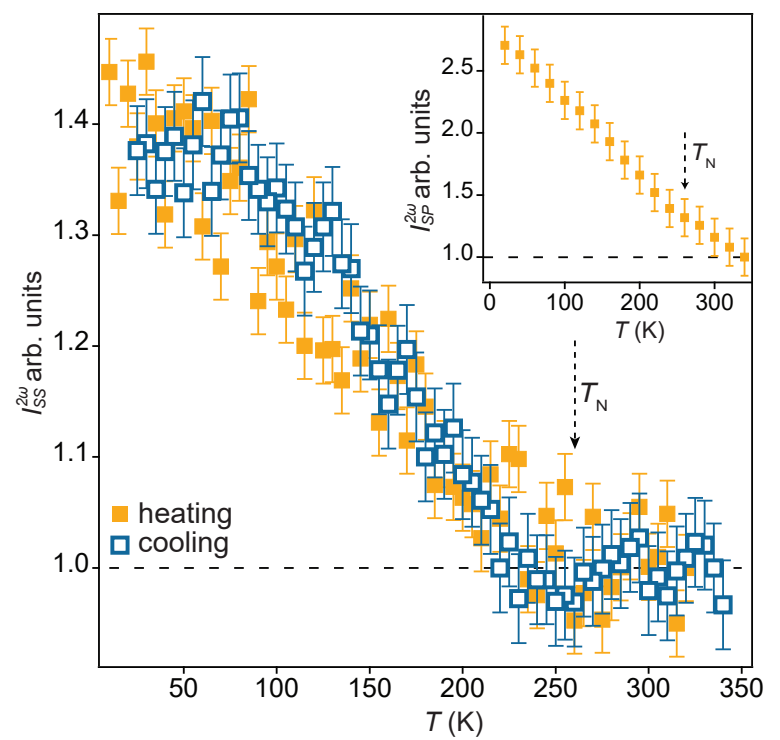

FIG. 3. SHG intensity measured in the $\mathrm{SS}$ channel at $\varphi=$ $3 \pi / 8$ upon heating (orange symbols) and cooling (blue symbols). Data are normalized to the $T=320 \mathrm{~K}$ value. Inset shows analogous data in the $\mathrm{SP}$ channel at $\varphi=\pi / 8$. Error bars represent the standard deviation over four independent measurements. See [19] for PP and PS data.

[Fig. 2(a)]. The absence of $x z$ and $y z$ mirror symmetries in the RA patterns [Fig. 2(h) \& (i)] therefore shows that the SHG response does not directly couple to the AFM order parameter $(\Psi)$. This is expected because neighboring $\mathrm{Cu}$ sites are structurally equivalent and related purely by time-reversal below $T_{\mathrm{N}}$, guaranteeing cancellation of any SHG process that is proportional to the local moment. It is possible for the SHG response to couple to $\Psi^{2}$ via magnetostriction [17, but the associated crystallographic point group is orthorhombic (point group $m m m$ ) and preserves the $x z$ and $y z$ mirror symmetries [19]. Averaging over $90^{\circ}$ rotated orthorhombic domains would not lift these symmetries in the RA-SHG patterns.

We first consider possible structural mechanisms for the observed symmetry breaking. The removal of vertical mirror planes lowers the crystallographic point group of $\mathrm{Sr}_{2} \mathrm{CuO}_{2} \mathrm{Cl}_{2}$ from $4 / \mathrm{mmm}$ to $4 / m$. This could arise, for example, through a staggered rotation and distortion of the $\mathrm{CuO}_{4} \mathrm{Cl}_{2}$ octahedra analogous to $\mathrm{Sr}_{2} \mathrm{IrO}_{4}$ [22]. However, x-ray and neutron diffraction studies find no evidence of such distortions either above or below $T_{\mathrm{N}}$ [11, 12, 21]. One can postulate that the $4 / m$ distortion only occurs at the surface. However, surface-sensitive Heatom scattering [23] and angle-resolved photoemission spectroscopy measurements 24] on $\mathrm{Sr}_{2} \mathrm{CuO}_{2} \mathrm{Cl}_{2}$ show no signs of (001) surface reconstruction or unit cell enlargement. Even if the mirror planes are somehow broken at the surface, it still cannot explain our data because ED SHG from the surface of a $4 / m$ structure is forbidden in the SS channel [19]. Moreover, it is unclear physically how an orthorhombic AFM order parameter with $m m m 1^{\prime}$ symmetry can induce a secondary structural order parameter with tetragonal $4 / m$ symmetry.

An alternative explanation is that the vertical mirror planes are broken by a hitherto undetected magnetic order parameter $(\Phi)$ that is coupled non-linearly to $\Psi$ and is described by a $4 / \mathrm{mm}^{\prime} \mathrm{m}^{\prime}$ magnetic point group. Here $m^{\prime}$ denotes a combined mirror and time-reversal operation. Lower symmetry subgroups of $4 / \mathrm{mm}^{\prime} \mathrm{m}^{\prime}$ cannot be ruled out but are not necessary to explain our data. A magnetic state with point group $4 / \mathrm{mm}^{\prime} \mathrm{m}^{\prime}$ has $A_{2 g}$ symmetry (inversion preserving) and transforms like the $z$ component of magnetization. Hence it is compatible with a ferroic ordering of odd-rank magnetic moments (dipole, octupole, etc [19]) along the $c$-axis and is consistent with a magneto-chiral state. Below, we survey the new SHG processes that can be induced by this magnetic state and interfere with the existing $4 / \mathrm{mmm}$ crystallographic EQ SHG signal.

One possibility is that the new SHG response couples linearly to $\Phi$. This can occur through an EQ process of the form $P_{i}^{2 \omega}=\chi_{i j k l}^{\mathrm{EQ}(c)} E_{j}^{\omega} \nabla_{k} E_{l}^{\omega}$ or a magnetic-dipole (MD) process of the form $P_{i}^{2 \omega}=\chi_{i j k}^{\mathrm{MD}(c)} E_{j}^{\omega} H_{k}^{\omega}$, where $E$ and $H$ are the electric and magnetic fields of the incident light at frequency $\omega, P$ is the induced electric polarization at the second harmonic, the $\chi$ 's are $c$-type (time-reversal odd) susceptibility tensors that are invariant under the symmetries of the $4 / \mathrm{mm}^{\prime} \mathrm{m}^{\prime}$ point group, and the $i, j, k$ indices run through the $x, y, z$ coordinates. Another possibility is that the new SHG response couples to $\Phi^{2}$ via magnetostriction, which would be governed by $i$-type (time-reversal even) EQ and MD susceptibility tensors respecting the $4 / m$ point group symmetries. We calculated expressions for the RA intensity in all of these cases [19]. Among the aforementioned processes, the $\chi^{\mathrm{MD}(c)}$ process stands out because $I_{\mathrm{PP}}^{2 \omega}=I_{\mathrm{SP}}^{2 \omega}=0$, $I_{\mathrm{PS}}^{2 \omega} \propto\left|\chi_{x z x}^{\mathrm{MD}(c)} \sin \theta\right|^{2}$ and $I_{\mathrm{SS}}^{2 \omega} \propto\left|\chi_{x x z}^{\mathrm{MD}(c)} \sin \theta\right|^{2}$. Attributing the SHG signal that onsets below $T_{\mathrm{N}}$ predominantly to this MD process would explain why we only observe mirror symmetry breaking below $T_{\mathrm{N}}$ in the Soutput channels and not the P-output channels [Figs 2 $\& 3]$. It is also consistent with the absence of any normal incidence SHG signal below $T_{\mathrm{N}}[19$.

The quasi-linear intensity increase in the P-output channels observed upon cooling [Fig. 3 inset] can be attributed to non-uniform changes in the crystallographic $\chi^{\mathrm{EQ}(i)}$ elements [25]. This likely arises from the anisotropic thermal contraction of the $\mathrm{Sr}_{2} \mathrm{CuO}_{2} \mathrm{Cl}_{2}$ lattice. As shown by neutron diffraction, between $T=300$ $\mathrm{K}$ and $25 \mathrm{~K}$ the $c$-axis contracts by $\sim 0.53 \%$ as compared to only $\sim 0.23 \%$ for the $a$-axis [12]. Therefore one expects a stronger temperature dependence for out-of-plane $(E \| c)$ compared to in-plane $(E \perp c)$ excitations. The fact that only the P-output channels are sensitive to out- 

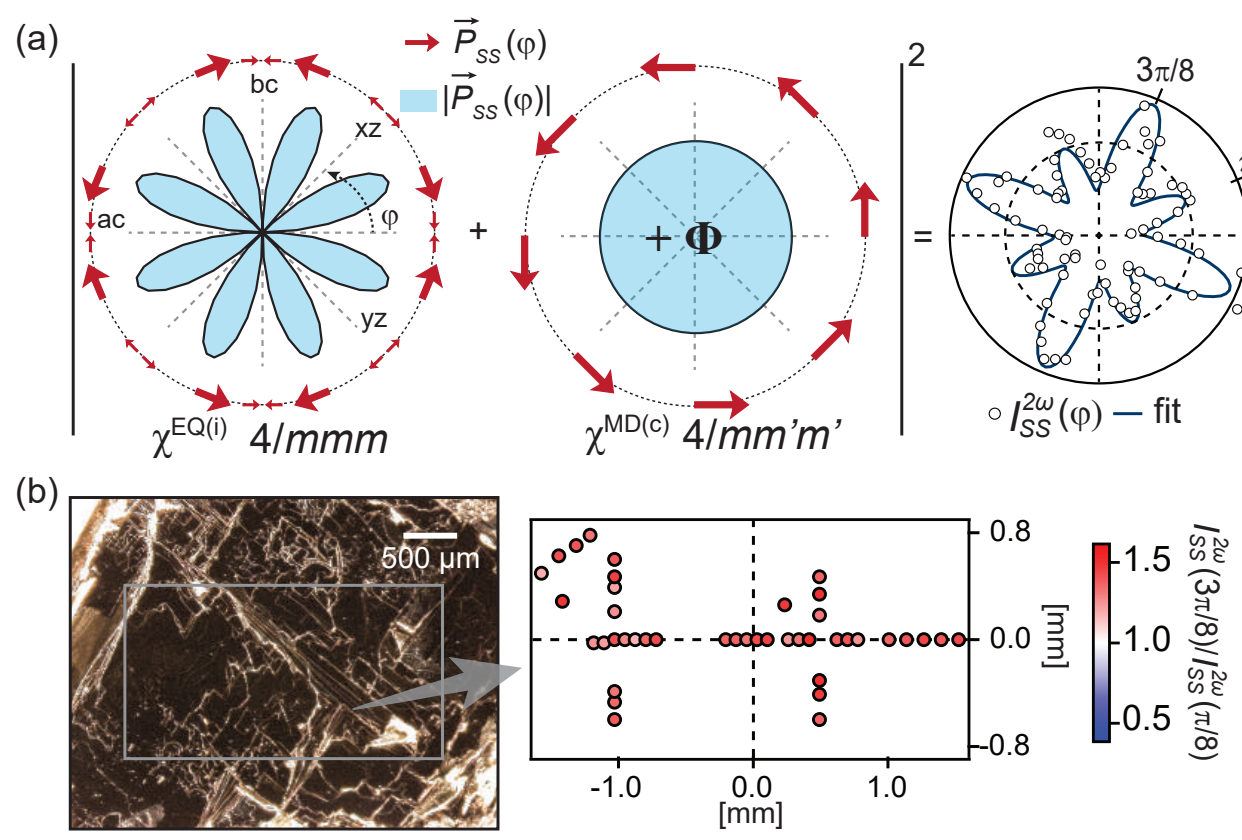

FIG. 4. (a) Illustration of how the low temperature RA pattern in the SS channel can be produced by a coherent superposition of $\chi^{\mathrm{EQ}(i)}(4 / \mathrm{mmm})$ and $\chi^{\mathrm{MD}(c)}\left(4 / \mathrm{mm}^{\prime} \mathrm{m}^{\prime}\right)$ processes. The red arrows denote the S-input light induced electric polarization projected along the S-output direction $\left(\vec{P}_{S S}\right)$ at each $\varphi$. The polar plots show the $\varphi$ dependence of $\left|\vec{P}_{S S}\right|$. A fit of this model (blue lines) to the RA data (circles) in the SS channel measured at $T=20 \mathrm{~K}$ is shown on the right. The best fit was obtained using an optical phase difference of $102^{\circ}$ between the EQ and MD processes. (b) Optical micrograph of the cleaved (001) surface of a $\mathrm{Sr}_{2} \mathrm{CuO}_{2} \mathrm{Cl}_{2}$ single crystal. RA-SHG patterns were measured in the SS channel at $T=80 \mathrm{~K}$ at the locations marked by colored circles within the gray box. The color scale encodes the intensity ratio between two adjacent lobes at $\varphi=3 \pi / 8$ and $\pi / 8$ extracted from fits [see panel (a)]. The slight spatial-dependence of this ratio is primarily due to systematic noise.

of-plane excitations (i.e. $\chi^{\mathrm{EQ}(i)}$ elements with $i, j$ or $l=z$ ) can explain why they exhibit the more marked temperature-dependent background [19].

Figure 4(a) illustrates how the observed symmetry breaking in the S-output channels can be generated by a new $\chi^{\mathrm{MD}(c)}$ contribution below $T_{\mathrm{N}}$. The SHG response above $T_{\mathrm{N}}$ is governed by an $i$-type EQ susceptibility tensor that respects $4 / \mathrm{mmm}$ symmetry and generates an RA pattern with eight lobes of equal intensity [Fig. 2(d) \& (e)]. Below $T_{\mathrm{N}}$ a $c$-type MD susceptibility tensor that respects $4 / \mathrm{mm}^{\prime} \mathrm{m}^{\prime}$ symmetry emerges and generates a $\varphi$-independent contribution. Coherent superposition of these two processes yields oblique incidence RA intensities proportional to $|\alpha \sin 4 \varphi+\beta|^{2}$, where $\alpha=\chi_{x y x y}^{\mathrm{EQ}(i)}-\chi_{x x x x}^{\mathrm{EQ}(i)}+2 \chi_{x x y y}^{\mathrm{EQ}(i)}$ and $\beta=\chi_{x z x}^{\mathrm{MD}(c)}$ or $\chi_{x x z}^{\mathrm{MD}(c)}$ for the PS and SS channels respectively. We note that in an absorbing medium, the tensor elements are generally complex. This allows interference between the EQ and $\mathrm{MD}$ contributions, resulting in an alternating lobe intensity and a lifting of nodes that is consistent with observations.

Finally we discuss the microscopic origins of $\Phi$. The simplest magnetic state with $A_{2 g}$ symmetry is a $c$-axis oriented ferromagnet, which can arise from uniform canting of the $\mathrm{Cu}$ spins out of the $a b$ plane. But x-ray mag- netic circular dichroism measurements on $\mathrm{Sr}_{2} \mathrm{CuO}_{2} \mathrm{Cl}_{2}$ detect no out-of-plane spin canting [26], in keeping with its reported structure that forbids DM interactions. Surface spin canting also seems unlikely given the absence of surface reconstruction [23, 24]. Furthermore, in the spontaneous spin canting scenario, one might expect to find a spatial distribution of time-reversed domains characterized by $\pm \Phi$ that can also flip upon thermal cycling across $T_{\mathrm{N}}$. Since $\chi_{x x z}^{\mathrm{MD}(c)}$ couples linearly to $\Phi$, a change in the sign of $\Phi$ should invert the intensity ratio of adjacent lobes in the S-output RA patterns. We collected low temperature RA patterns in the SS channel at multiple locations spanning millimeters across a sample using an optical spot size of $\sim 40 \mu \mathrm{m}$. We deliberately selected a cleaved surface with many terrace steps to search for both lateral and $c$-axis domains. Figure $4(\mathrm{~b})$ shows the intensity ratio of adjacent lobes $I_{\mathrm{SS}}^{2 \omega}(\varphi=3 \pi / 8) / I_{\mathrm{SS}}^{2 \omega}(\varphi=\pi / 8)$ as a function of position. Surprisingly, this ratio stays greater than 1 at all measured locations and following multiple thermal cycles across $T=320 \mathrm{~K}$ [19]. This indicates that $\Phi$ is locked to one sign, further arguing against a $\mathrm{Cu}$ spin canting scenario.

Instead, our results suggest that $\Phi$ may arise from an independent magnetic state that already exists above $T$ $=320 \mathrm{~K}$ and intrinsically forms large single domains, 
or has its orientation pinned by extrinsic effects such as correlated structural defects. The order parameter $\Phi$ is merely enhanced at $T_{\mathrm{N}}$ due to coupling to $\Psi$ so as to become more clearly detectable by SHG. Indeed, since $\Phi$ and $\Psi$ break independent symmetries, they are not constrained to have the same critical temperature and a coupling term of the form $\Phi^{2} \Psi^{2}$ is allowed by symmetry in the free energy. More complex intra-unit-cell spin or spin-lattice coupled arrangements with higher multipole moments [27, intra-unit-cell orbital loop current configurations 28 30] possibly associated with topological order [31, or long wavelength orbital magnetization density waves 32 that break vertical mirror planes are plausible candidates for $\Phi$. We note that the AFM form factor of $\mathrm{Sr}_{2} \mathrm{CuO}_{2} \mathrm{Cl}_{2}$ measured from neutron diffraction reportedly deviates from that of a free $\mathrm{Cu}^{2+}$ ion 33 . Resonant inelastic x-ray scattering measurements of its magnetic excitation spectrum also reveal the importance of further neighbor and four-spin ring exchange interactions [34, 35]. These data support the possibility of more exotic orders beyond the classical AFM state. Regardless of microscopic origin, the existence of $\Phi$ may be connected to several unexplained properties of the cuprate pseudogap state including a large chirality-induced thermal Hall conductivity [6, 8, (allowed by $4 / \mathrm{mm}^{\prime} \mathrm{m}^{\prime}$ but not $m m m 1^{\prime}$ [36]), the enhancement of vertical mirror symmetry breaking observed by $\mathrm{THz}$ and SHG polarimetry [19, 37, 38, and a polar Kerr rotation (allowed by $4 / \mathrm{mm}^{\prime} \mathrm{m}^{\prime}$ ) that can be magnetic field trained well above the pseudogap temperature [39].

\section{ACKNOWLEDGEMENTS}

We acknowledge helpful conversations with Dante Kennes, Steve Kivelson, Patrick Lee, Olexei Motrunich, Damjan Pelc and Kemp Plumb. We also thank Louis Taillefer and Gaël Grissonnanche for sharing unpublished data. The SHG work is supported by an ARO PECASE award W911NF-17-1-0204. D.H. also acknowledges support for instrumentation from the David and Lucile Packard Foundation and from the Institute for Quantum Information and Matter (IQIM), an NSF Physics Frontiers Center (PHY-1733907). A.d.l.T. acknowledges support from the Swiss National Science Foundation through an Early Postdoc Mobility Fellowship (P2GEP2_165044). K.L.S. acknowledges a Caltech Prize Postdoctoral Fellowship. S.S. acknowledges support from NSF grant DMR-2002850. M.S.S. acknowledges support from the German National Academy of Sciences Leopoldina through Grant LPDS 2016-12. M.R.N. was supported by the Materials Sciences and Engineering Division, Basic Energy Sciences, Office of Science, U.S. Department of Energy. The work at the University of Minnesota was funded by the U.S. Department of Energy through the University of Minnesota Center for Quantum Materials, under Grant No. DE-SC-0016371.

\section{METHODS}

\section{Single crystal growth and preparation}

Single crystals of $\mathrm{Sr}_{2} \mathrm{CuO}_{2} \mathrm{Cl}_{2}$ were grown by standard methods described in Ref. 12. Crystals were pre-aligned using x-ray Laue diffraction, cleaved along their (001) surface in a $\mathrm{N}_{2}$-rich environment to avoid air exposure, and then immediately pumped down to pressures below $10^{-7}$ torr.

\section{Second harmonic generation measurements}

The RA-SHG experiments were performed using a high-speed rotating scattering plane based setup [18. Incident laser light was delivered by a Ti:sapphire regenerative amplifier (pulse width $80 \mathrm{fs}$, repetition rate $100 \mathrm{kHz}$, center wavelength $800 \mathrm{~nm}$ ) seeding an optical parametric amplifier. The light was focused to a spot size of $\sim 40 \mu \mathrm{m}$ onto optically flat regions of the crystals. The infrared photon energies used in this study are below the charge gap of $\mathrm{Sr}_{2} \mathrm{CuO}_{2} \mathrm{Cl}_{2}$ and very weakly absorbed [40]. Therefore a minimum fluence of around $3 \mathrm{~mJ} / \mathrm{cm}^{2}$ was necessary to acquire RA-SHG patterns of reasonable quality using obliquely incident $800 \mathrm{~nm}$ light. All data presented were reproduced on multiple spots across multiple single crystals.

[1] B. Keimer, S. A. Kivelson, M. R. Norman, S. Uchida, and J. Zaanen, Nature 518, 179 (2015)

[2] M. R. Norman, D. Pines, and C. Kallin, Advances in Physics 54, 715 (2005).

[3] E. Fradkin, S. A. Kivelson, and J. M. Tranquada, Rev. Mod. Phys. 87, 457 (2015)

[4] C. Proust and L. Taillefer, Annual Review of Condensed Matter Physics 10, 409 (2019)

[5] P. A. Lee, N. Nagaosa, and X.-G. Wen, Rev. Mod. Phys. 78, 17 (2006).

[6] G. Grissonnanche, A. Legros, S. Badoux, E. Lefrançois, V. Zatko, M. Lizaire, F. Laliberté, A. Gourgout, J.S. Zhou, S. Pyon, T. Takayama, H. Takagi, S. Ono, N. Doiron-Leyraud, and L. Taillefer, Nature 571, 376 (2019)

[7] G. Grissonnanche, S. Thériault, A. Gourgout, M.-E. Boulanger, E. Lefrançois, A. Ataei, F. Laliberté, M. Dion, J.-S. Zhou, S. Pyon, T. Takayama, H. Takagi, N. DoironLeyraud, and L. Taillefer, Nature Physics (2020), 10.1038/s41567-020-0965-y

[8] M.-E. Boulanger, G. Grissonnanche, S. Badoux, A. Allaire, E. Lefranois, A. Legros, A. Gourgout, M. Dion, C. H. Wang, X. H. Chen, R. Liang, W. N. Hardy, D. A. 
Bonn, and L. Taillefer, (2020), arXiv:2007.05088 [condmat.str-el]

[9] J. H. Han, J.-H. Park, and P. A. Lee, Phys. Rev. B 99, 205157 (2019)

[10] R. Samajdar, S. Chatterjee, S. Sachdev, and M. S. Scheurer, Phys. Rev. B 99, 165126 (2019).

[11] B. Grande and H. Müller-Buschbaum, Z. Anorg. Allg. Chem. 417, 68 (1975).

[12] L. L. Miller, X. L. Wang, S. X. Wang, C. Stassis, D. C. Johnston, J. Faber, and C.-K. Loong, Phys. Rev. B 41, 1921 (1990)

[13] A. Cuccoli, T. Roscilde, R. Vaia, and P. Verrucchi, Phys. Rev. Lett. 90, 167205 (2003).

[14] B. J. Suh, F. Borsa, L. L. Miller, D. C. Johnston, D. R. Torgeson, and M. Corti, J. Appl. Phys. 79, 5084 (1996).

[15] K. Katsumata, M. Hagiwara, Z. Honda, J. Satooka, A. Aharony, R. J. Birgeneau, F. C. Chou, O. EntinWohlman, A. B. Harris, M. A. Kastner, Y. J. Kim, and Y. S. Lee, Europhys. Lett. 54, 508 (2001)

[16] M. Greven, R. J. Birgeneau, Y. Endoh, M. A. Kastner, M. Matsuda, and G. Shirane, Zeitschrift für Physik B Condensed Matter 96, 465 (1995)

[17] M. Fiebig, V. V. Pavlov, and R. V. Pisarev, J. Opt. Soc. Am. B 22, 96 (2005)

[18] J. W. Harter, L. Niu, A. J. Woss, and D. Hsieh, Opt. Lett. 40, 4671 (2015)

[19] "See supplementary information,".

[20] H. S. Choi, Y. S. Lee, T. W. Noh, E. J. Choi, Y. Bang, and Y. J. Kim, Phys. Rev. B 60, 4646 (1999)

[21] D. Vaknin, S. K. Sinha, C. Stassis, L. L. Miller, and D. C. Johnston, Phys. Rev. B 41, 1926 (1990)

[22] F. Ye, X. Wang, C. Hoffmann, J. Wang, S. Chi, M. Matsuda, B. C. Chakoumakos, J. A. Fernandez-Baca, and G. Cao, Phys. Rev. B 92, 201112(R) (2015)

[23] M. Farzaneh, X. F. Liu, M. El-Batanouny, and F. C. Chou, Phys. Rev. B 72, 085409 (2005).

[24] C. Dürr, S. Legner, R. Hayn, S. V. Borisenko, Z. Hu, A. Theresiak, M. Knupfer, M. S. Golden, J. Fink, F. Ronning, Z.-X. Shen, H. Eisaki, S. Uchida, C. Janowitz, R. Müller, R. L. Johnson, K. Rossnagel, L. Kipp, and G. Reichardt, Phys. Rev. B 63, 014505 (2000).

[25] A. Ron, E. Zoghlin, L. Balents, S. D. Wilson, and D. Hsieh, Nature Comm. 10, 1654 (2019)

[26] G. M. De Luca, G. Ghiringhelli, M. Moretti Sala, S. Di Matteo, M. W. Haverkort, H. Berger, V. Bisogni, J. C. Cezar, N. B. Brookes, and M. Salluzzo, Phys. Rev. B 82, 214504 (2010).

[27] M. Fechner, M. J. A. Fierz, F. Thöle, U. Staub, and N. A. Spaldin, Phys. Rev. B 93, 174419 (2016)

[28] C. Varma, Phys. Rev. B 55, 14554 (1997).

[29] Y. He, P. A. Lee, and C. M. Varma, Phys. Rev. B 89, 035119 (2014)

[30] M. S. Scheurer and S. Sachdev, Phys. Rev. B 98, 235126 (2018)

[31] S. Sachdev, Rev. Mod. Phys. 75, 913 (2003).

[32] Z. Dai, T. Senthil, and P. A. Lee, Phys. Rev. B 101, $064502(2020)$

[33] X. L. Wang, L. L. Miller, J. Ye, C. Stassis, B. N. Harmon, D. C. Johnston, A. J. Schultz, and C. K. Loong, J. Appl. Phys. 67, 4524 (1990)

[34] M. Guarise, B. Dalla Piazza, M. Moretti Sala, G. Ghiringhelli, L. Braicovich, H. Berger, J. N. Hancock, D. van der Marel, T. Schmitt, V. N. Strocov, L. J. P. Ament, J. van den Brink, P.-H. Lin, P. Xu, H. M.
Rønnow, and M. Grioni, Phys. Rev. Lett. 105, 157006 (2010).

[35] K. W. Plumb, A. T. Savici, G. E. Granroth, F. C. Chou, and Y.-J. Kim, Phys. Rev. B 89, 180410 (2014)

[36] M. Seemann, D. Ködderitzsch, S. Wimmer, and H. Ebert, Phys. Rev. B 92, 155138 (2015).

[37] Y. Lubashevsky, L. Pan, T. Kirzhner, G. Koren, and N. P. Armitage, Phys. Rev. Lett. 112, 147001 (2014).

[38] L. Zhao, C. A. Belvin, R. Liang, D. A. Bonn, W. N. Hardy, N. P. Armitage, and D. Hsieh, Nature Phys. 13, 250 (2017)

[39] J. Xia, E. Schemm, G. Deutscher, S. A. Kivelson, D. A. Bonn, W. N. Hardy, R. Liang, W. Siemons, G. Koster, M. M. Fejer, and A. Kapitulnik, Phys. Rev. Lett. 100, $127002(2008)$.

[40] J. D. Perkins, J. M. Graybeal, M. A. Kastner, R. J. Birgeneau, J. P. Falck, and M. Greven, Phys. Rev. Lett. 71, 1621 (1993) 


\title{
SUPPLEMENTARY INFORMATION \\ Anomalous mirror symmetry breaking in a model insulating cuprate $\mathrm{Sr}_{2} \mathrm{CuO}_{2} \mathrm{Cl}_{2}$
}

\author{
A. de la Torre, ${ }^{1,2}$ K. L. Seyler, ${ }^{1,2}$ L. Zhao, ${ }^{3}$ S. Di Matteo, ${ }^{4}$ M. S. Scheurer, ${ }^{5}$ Y. Li, ${ }^{6}$ B. Yu, ${ }^{6}$ \\ M. Greven, ${ }^{6}$ S. Sachdev, ${ }^{5}$ M. R. Norman, ${ }^{7}$ and D. Hsieh, ${ }^{1,2}$
}

\section{CONTENTS}

1. Mathematical expressions for SHG processes

2. Comparison to GaAs

3. Simulated RA-SHG patterns using AFM point group

4. Description of possible $A_{2 \mathrm{~g}}$ order parameters

5. Temperature dependence of normal incidence intensity

6. Temperature dependence of intensity in PS and PP channels

7. Temperature and $\varphi$-dependence of intensity in SP and PP channels

8. Effects of non-uniform lattice contraction

9. Laser fluence and average power considerations

10. Effects of thermal cycling

11. Discussion of the effects of crystal imperfections

12. Comparison to prior SHG work on YBCO 


\section{Mathematical expressions for SHG processes}

Table S1 summarizes all of the SHG processes discussed in our work and our physical motivation for considering them. In addition, we also included MD and EQ SHG from the inversion preserving

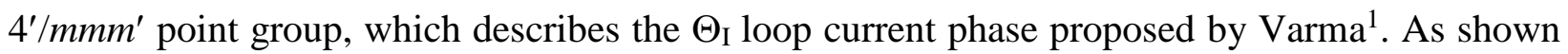
in Table S2, this is not consistent with our data. Note that ED, MD and EQ refer to the following processes respectively: $P_{i}^{2 \omega}=\chi_{i j k}^{E D} E_{j}^{\omega} E_{k}^{\omega}, P_{i}^{2 \omega}=\chi_{i j k}^{M D} E_{j}^{\omega} H_{k}^{\omega}$ and $P_{i}^{2 \omega}=\chi_{i j k l}^{E Q} E_{j}^{\omega} \nabla_{k} E_{l}^{\omega}$. The structure of the SHG susceptibility tensor associated with each process and point group is represented by a letter followed by a number (e.g. H3), in accordance with the nomenclature used by Birss (Ref. 2). Explicit forms of these tensors are listed later on, together with expressions for the RA-SHG pattern calculated in all four polarization channels PP, SP, PS and SS.

Table S1: Summary of SHG processes considered in this work.

\begin{tabular}{|c|c|c|c|c|}
\hline Point group & ED & MD & EQ & Physical origin \\
\hline $4 / \mathrm{mmm}$ & --- & H3 & $H 4$ & Reported crystal structure \\
\hline $4 m m$ & I3 & Not shown & Not shown & Surface of $4 / \mathrm{mmm}$ \\
\hline$m m m 1^{\prime}$ & --- & D3 & $D 4$ & Reported AFM structure \\
\hline$m m 21^{\prime}$ & E3 & Not shown & Not shown & Surface of $m m m 1^{\prime}$ \\
\hline $4 / m$ & --- & F3 & F4 & Candidate structure for $T<T_{\mathrm{N}}$ \\
\hline $4 / \mathrm{mm}^{\prime} \mathrm{m}^{\prime}$ & --- & HЗ/ІЗ(c) & $H 4 / I 4(c)$ & $A_{2 g}$ magnetic order \\
\hline $4^{\prime} / \mathrm{mmm}^{\prime}$ & --- & H3/J3(c) & $H 4 / J 4(c)$ & Varma $\Theta_{\text {I }}$ loop current order ${ }^{1}$ \\
\hline
\end{tabular}

For magnetic point groups, unless indicated, $c$-type (time-reversal odd) and $i$-type (time-reversal even) tensors have the same expressions. The symbol --- means the process is forbidden. For inversion broken point groups, we only consider the dominant ED contribution. 
Table S2 summarizes the dependence of the SHG intensity on the incident angle $\theta$ and scattering plane angle $\varphi$ for all considered processes and point groups in each of the four polarization channels. Importantly, only the $\mathrm{MD}(\mathrm{c})$ process from a $4 / \mathrm{mm}^{\prime} \mathrm{m}^{\prime}$ point group has a forbidden response in the PS and SS channels, which is necessary to explain our data. We also note that the clear $\theta$-dependence we observe in the SS channel immediately rules out an ED process.

Therefore no bulk inversion broken point groups are considered.

Table S2: Summary of angular dependences of SHG processes

\begin{tabular}{|c|c|c|c|c|c|}
\hline Point group & Process & PP & SP & PS & SS \\
\hline 4/mmm & MD & $\theta$ & $\theta$ & --- & --- \\
\hline 4/mmm & EQ & $\theta, \varphi$ & $\theta, \varphi$ & $\theta, \varphi$ & $\theta, \varphi$ \\
\hline $4 m m$ & ED & $\theta$ & $\theta$ & --- & --- \\
\hline$m m m 1^{\prime}$ & $\operatorname{MD}(i / c)$ & $\theta, \varphi$ & $\theta, \varphi$ & $\theta, \varphi$ & $\theta, \varphi$ \\
\hline$m m m 1^{\prime}$ & $\operatorname{EQ}(i / c)$ & $\theta, \varphi$ & $\theta, \varphi$ & $\theta, \varphi$ & $\theta, \varphi$ \\
\hline$m m 21^{\prime}$ & $\operatorname{ED}(i / c)$ & $\theta, \varphi$ & $\theta, \varphi$ & $\theta, \varphi$ & -- \\
\hline $4 / m$ & $\mathrm{MD}$ & $\theta$ & $\theta$ & $\theta$ & $\theta$ \\
\hline $4 / m$ & EQ & $\theta, \varphi$ & $\theta, \varphi$ & $\theta, \varphi$ & $\theta, \varphi$ \\
\hline $4 / m^{\prime} m^{\prime}$ & $\mathrm{MD}(c)$ & --- & --- & $\theta$ & $\theta$ \\
\hline $4 / \mathrm{mm}^{\prime} m^{\prime}$ & $\mathrm{EQ}(c)$ & $\theta, \varphi$ & $\theta, \varphi$ & $\theta, \varphi$ & $\theta, \varphi$ \\
\hline $4^{\prime} / \mathrm{mmm}^{\prime}$ & $\mathrm{MD}(c)$ & $\theta, \varphi$ & $\theta, \varphi$ & $\theta, \varphi$ & $\theta, \varphi$ \\
\hline $4^{\prime} / \mathrm{mmm}^{\prime}$ & $\mathrm{EQ}(c)$ & $\theta, \varphi$ & $\theta, \varphi$ & $\theta, \varphi$ & $\theta, \varphi$ \\
\hline
\end{tabular}

Symbol definitions: $(\theta, \varphi)$ means depends on both $\theta$ and $\varphi,(\theta)$ means depends only on $\theta$, (---) means forbidden. Detailed dependences on $\theta$ and $\varphi$ are provided below in this section. 


\section{ED tensors}

\section{E3, $\left\{\boldsymbol{m m} 21^{\prime}\right\}$}

$$
\chi_{i j k}^{E D}=\left(\begin{array}{ccc}
\left(\begin{array}{c}
0 \\
0 \\
x x z
\end{array}\right) & \left(\begin{array}{c}
0 \\
0 \\
0
\end{array}\right) & \left(\begin{array}{c}
x x z \\
0 \\
0
\end{array}\right) \\
\left(\begin{array}{l}
0 \\
0 \\
0
\end{array}\right) & \left(\begin{array}{c}
0 \\
0 \\
y y z
\end{array}\right) & \left(\begin{array}{c}
0 \\
y y z \\
0
\end{array}\right) \\
\left(\begin{array}{c}
z x x \\
0 \\
0
\end{array}\right) & \left(\begin{array}{c}
0 \\
z y y \\
0
\end{array}\right) & \left(\begin{array}{c}
0 \\
0 \\
z z z
\end{array}\right)
\end{array}\right)
$$

$$
\begin{aligned}
& \boldsymbol{I}^{P P}(\mathbf{2} \boldsymbol{\omega}) \sim\left(\chi_{z z z} \sin ^{3}(\theta)+\sin (\theta) \cos ^{2}(\theta)\left(\left(-2 \chi_{x x z}+\chi_{z x x}\right) \cos ^{2}(\boldsymbol{\varphi})+\left(-2 \chi_{y y z}+\right.\right.\right. \\
& \left.\left.\left.\chi_{z y y}\right) \sin ^{2}(\boldsymbol{\varphi})\right)\right)^{2} \\
& \boldsymbol{I}^{P S}(\mathbf{2} \boldsymbol{\omega}) \sim 4 \sin ^{2}(\theta) \cos ^{2}(\theta)\left(\left(\chi_{x x z}-\chi_{y y z}\right) \sin (\boldsymbol{\varphi}) \cos (\boldsymbol{\varphi})\right)^{2} \\
& \boldsymbol{I}^{\boldsymbol{S P}}(\mathbf{2} \boldsymbol{\omega}) \sim \sin ^{2}(\theta)\left(\chi_{z x x} \sin ^{2}(\boldsymbol{\varphi})+\chi_{z y y} \cos ^{2}(\boldsymbol{\varphi})\right)^{2} \\
& \boldsymbol{I}^{S \boldsymbol{S}}(\mathbf{2} \boldsymbol{\omega})=0
\end{aligned}
$$

\section{$I 3,\{4 \mathbf{m m}\}$}

$$
\chi_{i j k}^{E D}=\left(\begin{array}{ccc}
\left(\begin{array}{c}
0 \\
0 \\
\mathrm{xxz}
\end{array}\right) & \left(\begin{array}{c}
0 \\
0 \\
0
\end{array}\right) & \left(\begin{array}{c}
\mathrm{xxz} \\
0 \\
0
\end{array}\right) \\
\left(\begin{array}{c}
0 \\
0 \\
0
\end{array}\right) & \left(\begin{array}{c}
0 \\
0 \\
\mathrm{xxz}
\end{array}\right) & \left(\begin{array}{c}
0 \\
\mathrm{xxz} \\
0
\end{array}\right) \\
\left(\begin{array}{c}
\mathrm{ZXX} \\
0 \\
0
\end{array}\right) & \left(\begin{array}{c}
0 \\
\mathrm{zxx} \\
0
\end{array}\right) & \left(\begin{array}{c}
0 \\
0 \\
\mathrm{zzz}
\end{array}\right)
\end{array}\right)
$$

$$
\begin{aligned}
& \boldsymbol{I}^{P P}(\mathbf{2} \boldsymbol{\omega}) \sim\left[\sin ^{2}(\theta)\left(\left(-2 \chi_{\mathrm{xxz}}+\chi_{\mathrm{zxx}}\right) \cos ^{2}(\theta)-\chi_{\mathrm{zzz}} \sin (\theta)\right)^{2}\right] \\
& \boldsymbol{I}^{P S}(\mathbf{2} \boldsymbol{\omega})=0 \\
& \boldsymbol{I}^{S P}(\mathbf{2} \boldsymbol{\omega}) \sim\left[\chi_{\mathrm{zxx}}{ }^{2} \sin ^{2}(\theta)\right] \\
& \boldsymbol{I}^{S S}(\mathbf{2} \boldsymbol{\omega})=0
\end{aligned}
$$




\section{MD tensors}

D3, $\left\{\boldsymbol{m m m} \mathbf{1}^{\prime}\right\}$

$$
\begin{aligned}
& \chi_{i j k}^{M D}=\left(\begin{array}{ccc}
\left(\begin{array}{l}
0 \\
0 \\
0
\end{array}\right) & \left(\begin{array}{c}
0 \\
0 \\
\mathrm{xyz}
\end{array}\right) & \left(\begin{array}{c}
0 \\
\mathrm{xzy} \\
0
\end{array}\right) \\
\left(\begin{array}{c}
0 \\
0 \\
\mathrm{yxz}
\end{array}\right) & \left(\begin{array}{l}
0 \\
0 \\
0
\end{array}\right) & \left(\begin{array}{c}
\mathrm{yzx} \\
0 \\
0
\end{array}\right) \\
\left(\begin{array}{c}
0 \\
\mathrm{zxy} \\
0
\end{array}\right) & \left(\begin{array}{c}
\mathrm{zyx} \\
0 \\
0
\end{array}\right) & \left(\begin{array}{l}
0 \\
0 \\
0
\end{array}\right)
\end{array}\right) \\
& \boldsymbol{I}^{\boldsymbol{P P}}(2 \boldsymbol{\omega}) \sim\left[\sin ^{2}(2 \theta)\left(\left(\chi_{\mathrm{xyz}}-\chi_{\mathrm{zyx}}\right) \cos ^{2}(\boldsymbol{\varphi})+\left(-\chi_{\mathrm{yxz}}+\chi_{\mathrm{zxy}}\right) \sin ^{2}(\boldsymbol{\varphi})\right)^{2}\right] \\
& I^{P S}(2 \omega) \sim\left[\left(\chi_{\mathrm{xyz}}+\chi_{\mathrm{yxz}}\right)^{2} \cos ^{2}(\varphi) \sin ^{2}(\varphi) \sin ^{2}(\theta)\right] \\
& I^{S P}(\mathbf{2} \boldsymbol{\omega}) \sim\left[\left(\left(-\chi_{\mathrm{xyz}}+\chi_{\mathrm{zyx}}\right) \cos ^{2}(\boldsymbol{\varphi})+\left(\chi_{\mathrm{yxz}}-\chi_{\mathrm{zxy}}\right) \sin ^{2}(\boldsymbol{\varphi})\right)^{2} \cos ^{2}(\theta) \sin ^{2}(\theta)\right] \\
& I^{S S}(2 \omega) \sim\left[\left(\chi_{\mathrm{xyz}}+\chi_{\mathrm{yxz}}\right)^{2} \cos ^{2}(\varphi) \sin ^{2}(\varphi) \sin ^{2}(\theta)\right]
\end{aligned}
$$

F3, $\{\mathbf{4} / \mathbf{m}\}$

$$
\chi_{i j k}^{M D}=\left(\begin{array}{ccc}
\left(\begin{array}{c}
0 \\
0 \\
\mathrm{xxz}
\end{array}\right) & \left(\begin{array}{c}
0 \\
0 \\
\mathrm{xyz}
\end{array}\right) & \left(\begin{array}{c}
\mathrm{xzx} \\
\mathrm{xzy} \\
0
\end{array}\right) \\
\left(\begin{array}{c}
0 \\
0 \\
-\mathrm{xyz}
\end{array}\right) & \left(\begin{array}{c}
0 \\
0 \\
\mathrm{xxz}
\end{array}\right) & \left(\begin{array}{c}
-\mathrm{xzy} \\
\mathrm{xzx} \\
0
\end{array}\right) \\
\left(\begin{array}{c}
\mathrm{zxx} \\
\mathrm{zxy} \\
0
\end{array}\right) & \left(\begin{array}{c}
-\mathrm{zxy} \\
\mathrm{zxx} \\
0
\end{array}\right) & \left(\begin{array}{c}
0 \\
0 \\
\mathrm{zzz}
\end{array}\right)
\end{array}\right)
$$

$$
\begin{aligned}
& \boldsymbol{I}^{P P}(2 \boldsymbol{\omega}) \sim\left[\left(\chi_{\mathrm{xyz}}-\chi_{\mathrm{zxy}}\right)^{2} \cos ^{2}(\theta) \sin ^{2}(\theta)\right] \\
& \boldsymbol{I}^{\boldsymbol{P S}}(\mathbf{2} \boldsymbol{\omega}) \sim\left[\chi_{\mathrm{xzx}} \sin ^{2}(\theta)\right] \\
& \boldsymbol{I}^{\boldsymbol{S P}}(\mathbf{2} \boldsymbol{\omega}) \sim\left[\left(\chi_{\mathrm{xyz}}+\chi_{\mathrm{zxy}}\right)^{2} \cos ^{2}(\theta) \sin ^{2}(\theta)\right] \\
& \boldsymbol{I}^{S \boldsymbol{S}}(\mathbf{2} \boldsymbol{\omega}) \sim\left[\chi_{\mathrm{xxz}} \sin ^{2}(\theta)\right]
\end{aligned}
$$


H3, $\left\{4 / \mathbf{m m m}, \mathbf{4} / \mathbf{m m}^{\prime} \mathbf{m}^{\prime}(\mathrm{i}), \mathbf{4}^{\prime} / \mathbf{m m m}^{\prime}\right.$ (i) $\}$

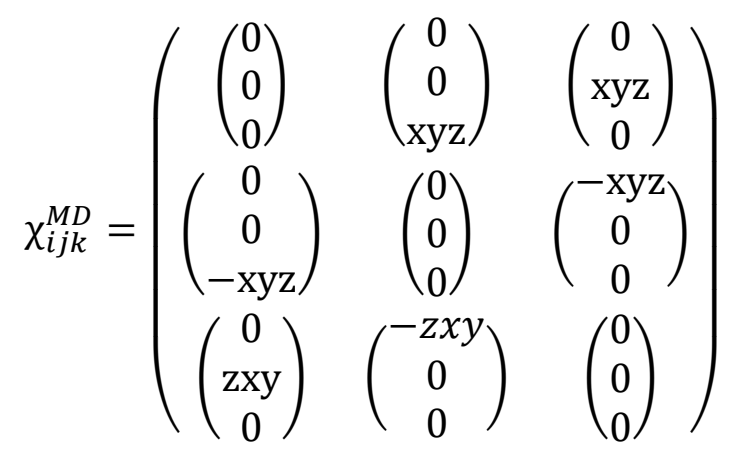

$$
\begin{aligned}
& \boldsymbol{I}^{\boldsymbol{P P}}(2 \boldsymbol{\omega}) \sim\left[\left(\chi_{\mathrm{xyz}}-\chi_{\mathrm{zxy}}\right)^{2} \cos ^{2}(\theta) \sin ^{2}(\theta)\right] \\
& I^{P S}(2 \omega) \sim 0 \\
& \boldsymbol{I}^{S P}(\mathbf{2} \boldsymbol{\omega}) \sim\left[\left(\chi_{\mathrm{xyz}}+\chi_{\mathrm{zxy}}\right)^{2} \cos ^{2}(\theta) \sin ^{2}(\theta)\right] \\
& I^{S S}(2 \omega) \sim 0
\end{aligned}
$$

\section{I3, $\left\{\mathbf{4} / \mathbf{m} \mathbf{m}^{\prime} \mathbf{m}^{\prime}(\boldsymbol{c})\right\}$}

$$
\chi_{i j k}^{M D}=\left(\begin{array}{ccc}
\left(\begin{array}{c}
0 \\
0 \\
\mathrm{xxz}
\end{array}\right) & \left(\begin{array}{c}
0 \\
0 \\
0
\end{array}\right) & \left(\begin{array}{c}
\mathrm{XZX} \\
0 \\
0
\end{array}\right) \\
\left(\begin{array}{c}
0 \\
0 \\
0
\end{array}\right) & \left(\begin{array}{c}
0 \\
0 \\
\mathrm{xxz}
\end{array}\right) & \left(\begin{array}{c}
0 \\
\mathrm{xzx} \\
0
\end{array}\right) \\
\left(\begin{array}{c}
\mathrm{ZxX} \\
0 \\
0
\end{array}\right) & \left(\begin{array}{c}
0 \\
\mathrm{zxx} \\
0
\end{array}\right) & \left(\begin{array}{c}
0 \\
0 \\
\mathrm{zzz}
\end{array}\right)
\end{array}\right)
$$

$$
\begin{aligned}
& I^{P P}(2 \boldsymbol{\omega}) \sim 0 \\
& I^{P S}(2 \boldsymbol{\omega}) \sim\left[\chi_{\mathrm{xzx}}{ }^{2} \sin ^{2}(\theta)\right] \\
& I^{S P}(2 \boldsymbol{\omega}) \sim 0 \\
& I^{S S}(2 \boldsymbol{\omega}) \sim\left[\chi_{\mathrm{xxz}}{ }^{2} \sin ^{2}(\theta)\right]
\end{aligned}
$$


J3, $\left\{\mathbf{4}^{\prime} / \mathbf{m m m}^{\prime}(\mathbf{c})\right\}$

$$
\chi_{i j k}^{M D}=\left(\begin{array}{ccc}
\left(\begin{array}{c}
0 \\
0 \\
0
\end{array}\right) & \left(\begin{array}{c}
0 \\
0 \\
\mathrm{xyz}
\end{array}\right) & \left(\begin{array}{c}
0 \\
\mathrm{xzy} \\
0
\end{array}\right) \\
\left(\begin{array}{c}
0 \\
0 \\
\mathrm{xyz}
\end{array}\right) & \left(\begin{array}{l}
0 \\
0 \\
0
\end{array}\right) & \left(\begin{array}{c}
\mathrm{xzy} \\
0 \\
0
\end{array}\right) \\
\left(\begin{array}{c}
0 \\
\mathrm{zx} y \\
0
\end{array}\right) & \left(\begin{array}{c}
z x y \\
0 \\
0
\end{array}\right) & \left(\begin{array}{l}
0 \\
0 \\
0
\end{array}\right)
\end{array}\right)
$$

$$
\begin{aligned}
I^{P P}(2 \omega) & \sim\left[\left(\chi_{\mathrm{xyz}}-\chi_{\mathrm{zxy}}\right)^{2} \cos ^{2}(2 \varphi) \sin ^{2}(2 \theta)\right] \\
\boldsymbol{I}^{P S}(2 \boldsymbol{\omega}) & \sim\left[\left(\chi_{\mathrm{xzy}}\right)^{2} \cos ^{2}(\theta) \sin ^{2}(2 \varphi)\right] \\
\boldsymbol{I}^{S P}(2 \boldsymbol{\omega}) & \sim\left[\left(\chi_{\mathrm{xyz}}-\chi_{\mathrm{zxy}}\right)^{2} \cos ^{2}(2 \varphi) \sin ^{2}(2 \theta)\right] \\
\boldsymbol{I}^{S S}(2 \boldsymbol{\omega}) & \sim\left[\left(\chi_{\mathrm{xyz}}\right)^{2} \cos ^{2}(\theta) \sin ^{2}(2 \varphi)\right]
\end{aligned}
$$




\section{$\underline{\text { EQ tensors }}$}

$D 4,\{\boldsymbol{m m m 1}\}$

$\chi_{i j k l}^{E Q}=\left(\begin{array}{ccc}\left(\begin{array}{ccc}\mathrm{xxxx} & 0 & 0 \\ 0 & \mathrm{xxyy} & 0 \\ 0 & 0 & \mathrm{xxzz}\end{array}\right) & \left(\begin{array}{ccc}0 & \mathrm{xyxy} & 0 \\ \mathrm{xxyy} & 0 & 0 \\ 0 & 0 & 0\end{array}\right) & \left(\begin{array}{ccc}0 & 0 & \mathrm{xzxz} \\ 0 & 0 & 0 \\ \mathrm{xxzz} & 0 & 0\end{array}\right) \\ \left(\begin{array}{ccc}0 & \mathrm{yxxy} & 0 \\ \mathrm{yxyx} & 0 & 0 \\ 0 & 0 & 0\end{array}\right) & \left(\begin{array}{ccc}\mathrm{yyxx} & 0 & 0 \\ 0 & \mathrm{yyyy} & 0 \\ 0 & 0 & \mathrm{yyzz}\end{array}\right) & \left(\begin{array}{ccc}0 & 0 & 0 \\ 0 & 0 & \mathrm{yzyz} \\ 0 & \mathrm{yyzz} & 0\end{array}\right) \\ \left(\begin{array}{ccc}0 & 0 & 0 \\ \mathrm{zxzx} & 0 & 0\end{array}\right) & \left(\begin{array}{ccc}0 & 0 & 0 \\ 0 & 0 & \mathrm{zzyy} \\ 0 & \mathrm{zyzy} & 0\end{array}\right) & \left(\begin{array}{ccc}\mathrm{zzxx} & 0 & 0 \\ 0 & \mathrm{zzyy} & 0 \\ 0 & 0 & \mathrm{zzzz}\end{array}\right)\end{array}\right)$

$$
\begin{aligned}
\boldsymbol{I}^{\boldsymbol{P P}}(\mathbf{2} \boldsymbol{\omega}) \sim[ & \cos (\theta) \sin ^{3}(\theta)\left(\chi_{z z z z}+\left(\chi_{x z x z}-2 \chi_{z z x x}\right) \cos ^{2}(\boldsymbol{\varphi})+\left(\chi_{y z y z}-2 \chi_{z z y y}\right) \sin ^{2}(\boldsymbol{\varphi})\right) \\
& -\sin (\theta) \cos ^{3}(\theta)\left(-\chi_{x x x x} \cos ^{4}(\boldsymbol{\varphi})\right. \\
& +\left(2 \chi_{x x z z}-\chi_{z x z x}\right. \\
& \left.-\left(2 \chi_{x x y y}+\chi_{x y x y}+\chi_{y x x y}+\chi_{y x y x}+\chi_{y y x x}\right) \sin ^{2}(\boldsymbol{\varphi})\right) \cos ^{2}(\boldsymbol{\varphi}) \\
& \left.\left.-\sin ^{2}(\boldsymbol{\varphi})\left(-2 \chi_{y y z z}+\chi_{z y z y}+\chi_{y y y y} \sin ^{2}(\boldsymbol{\varphi})\right)\right)^{2}\right]
\end{aligned}
$$$$
\boldsymbol{I}^{P \boldsymbol{S}}(\mathbf{2} \boldsymbol{\omega}) \sim\left[\operatorname { s i n } ( \theta ) \left(\left(-\chi_{x z x z}+\chi_{y z y z}\right) \sin ^{2}(\theta) \cos (\boldsymbol{\varphi}) \sin (\boldsymbol{\varphi})\right.\right.
$$$$
+\cos ^{2}(\theta)\left(\left(-\chi_{x x x x}+\chi_{y x x y}+\chi_{x y x y}+\chi_{y y x x}\right) \cos ^{3}(\varphi) \sin (\varphi)-\left(2 \chi_{x x y y}\right.\right.
$$$$
\left.\left.\left.\left.+\chi_{x y x y}-\chi_{y y y y}\right) \cos (\varphi) \sin ^{3}(\varphi)+\left(\chi_{x x z z}-\chi_{y y z z}\right) \sin (2 \varphi)\right)\right)^{2}\right]
$$

$$
\begin{gathered}
\boldsymbol{I}^{S \boldsymbol{P}}(\mathbf{2} \boldsymbol{\omega}) \sim\left[\operatorname { c o s } ^ { 2 } ( \theta ) \operatorname { s i n } ^ { 2 } ( \theta ) \left(\left(\chi_{x y x y} \cos ^{4}(\boldsymbol{\varphi})+\chi_{y x y x} \sin ^{4}(\boldsymbol{\varphi})\right.\right.\right. \\
\left.+\left(\chi_{x x x x}-2 \chi_{x x y y}-\chi_{y x x y}-\chi_{y y x x}+\chi_{y y y y}\right) \cos ^{2}(\boldsymbol{\varphi}) \sin ^{2}(\varphi)\right) \\
\left.\left.+\left(\chi_{z y z y} \cos ^{2}(\varphi)+\chi_{z x z x} \sin ^{2}(\boldsymbol{\varphi})\right)\right)^{2}\right]
\end{gathered}
$$

$$
\begin{aligned}
\boldsymbol{I}^{S S}(\mathbf{2} \boldsymbol{\omega}) \sim & {[(} \\
& \left(\chi_{x x x x}-2 \chi_{x x y y}-\chi_{y x y x}\right) \sin ^{2}(\boldsymbol{\varphi}) \\
& \left.\left.+\left(\chi_{x x x x}+\chi_{y y x x}+\chi_{y y x x}+\chi_{y y y y}\right) \cos ^{2}(\boldsymbol{\varphi})\right)^{2} \sin ^{2}(\theta) \cos ^{2}(\varphi) \sin ^{2}(\boldsymbol{\varphi})\right]
\end{aligned}
$$


$F 4,\{4 / m\}$

$$
\begin{aligned}
& \chi_{i j k l}^{E Q}
\end{aligned}
$$

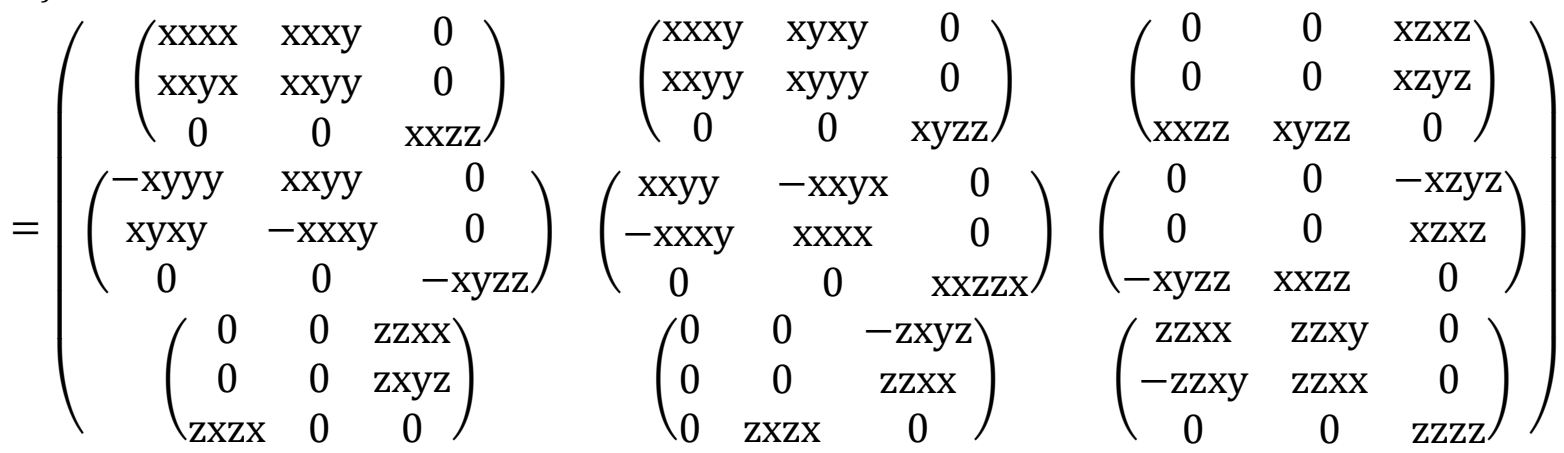

$$
\begin{aligned}
& \boldsymbol{I}^{\boldsymbol{P P}}(\mathbf{2} \boldsymbol{\omega}) \sim\left[\left(4\left(\chi_{\mathrm{zzzz}}-2 \chi_{\mathrm{zzxx}}+\chi_{\mathrm{xzxz}}\right) \cos (\theta) \sin ^{3}(\theta)\right.\right. \\
& +\sin (\theta) \cos ^{3}(\theta)\left(3 \chi_{\mathrm{xxxx}}+2 \chi_{\mathrm{xxyy}}-8 \chi_{\mathrm{xxzz}}+\chi_{\mathrm{xyxy}}+4 \chi_{\mathrm{zxzx}}\right. \\
& +\left(\chi_{\mathrm{xxxx}}-2 \chi_{\mathrm{xxyy}}-\chi_{\mathrm{xyxy}}\right) \cos (4 \varphi) \\
& \left.\left.\left.-\left(2 \chi_{\mathrm{xxxy}}-\chi_{\mathrm{xxyx}}+\chi_{\mathrm{xyyy}}\right) \sin (4 \varphi)\right)\right)^{2}\right] \\
& \boldsymbol{I}^{P \boldsymbol{S}}(\mathbf{2} \boldsymbol{\omega}) \sim\left[\left(4 \chi_{\mathrm{zzxy}} \sin ^{3}(\theta)\right.\right. \\
& +\cos ^{2}(\theta) \sin (\theta)\left(2 \chi_{\mathrm{xxxy}}+\chi_{\mathrm{xxyx}}+3 \chi_{\mathrm{xxyx}}-8 \chi_{\mathrm{xyzz}}\right. \\
& +\left(-2 \chi_{\mathrm{xxxy}}-\chi_{\mathrm{xxyx}}+\chi_{\mathrm{xyyy}}\right) \cos (4 \varphi) \\
& \left.\left.\left.+\left(-\chi_{\mathrm{xxxx}}+2 \chi_{\mathrm{xxxy}}+\chi_{\mathrm{xyxy}}\right) \sin (4 \varphi)\right)\right)^{2}\right] \\
& \boldsymbol{I}^{\boldsymbol{S P}}(\mathbf{2} \boldsymbol{\omega}) \sim\left[\left(-\chi_{\mathrm{zxzx}} \cos (\theta) \sin (\theta)\right.\right. \\
& -\frac{1}{4} \cos (\theta) \sin (\theta)\left(\chi_{\mathrm{xxxx}}-2 \chi_{\mathrm{xxyy}}+3 \chi_{\mathrm{xyxy}}\right. \\
& -\left(\chi_{\mathrm{xxxx}}-2 \chi_{\mathrm{xxyy}}-\chi_{\mathrm{xyxy}}\right) \cos (4 \varphi) \\
& \left.\left.\left.+\left(2 \chi_{\mathrm{xxxy}}+\chi_{\mathrm{xxyx}}-\chi_{\mathrm{xyyy}}\right) \sin (4 \varphi)\right)\right)^{2}\right] \\
& I^{S S}(\mathbf{2} \boldsymbol{\omega}) \sim\left[\operatorname { s i n } ^ { 2 } ( \theta ) \left(-2 \chi_{\mathrm{xxxy}}+3 \chi_{\mathrm{xxyx}}+\chi_{\mathrm{xyyy}}+\left(2 \chi_{\mathrm{xxxy}}+\chi_{\mathrm{xxyx}}-\chi_{\mathrm{xyyy}}\right) \cos (4 \varphi)\right.\right. \\
& \left.\left.+\left(\chi_{x x x x}-2 \chi_{x x y y}-\chi_{x y x y}\right) \sin (4 \varphi)\right)^{2}\right]
\end{aligned}
$$


$H 4,\left\{4 / \mathbf{m m m}, \mathbf{4 m m}, \mathbf{4} / \mathrm{mm}^{\prime} \mathrm{m}^{\prime}(\mathrm{i}), \mathbf{4}^{\prime} / \mathrm{mmm}^{\prime}(\mathrm{i})\right\}$

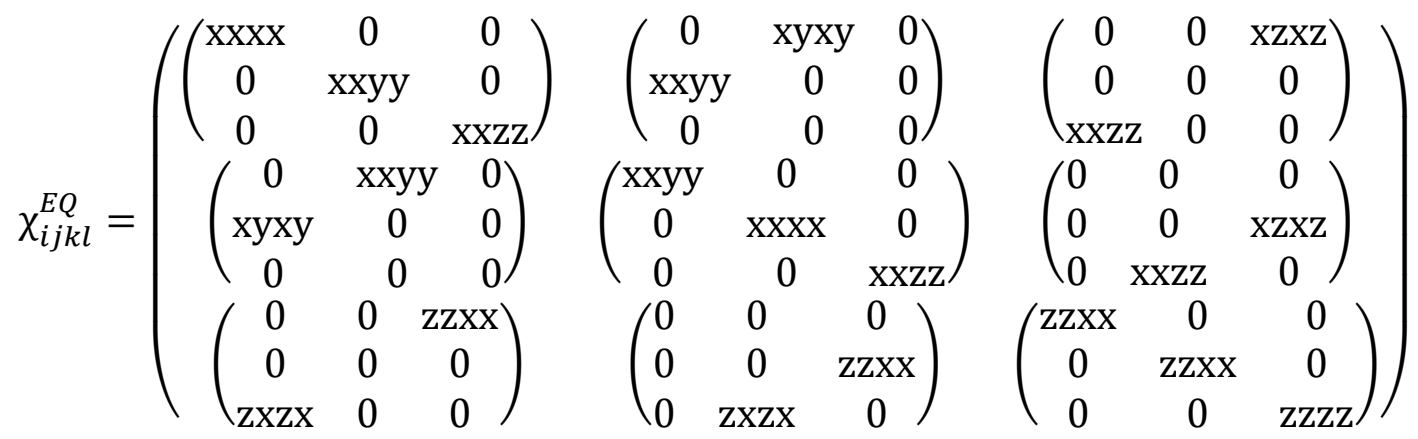

$$
\begin{aligned}
& \boldsymbol{I}^{\boldsymbol{P P}}(\mathbf{2} \boldsymbol{\omega}) \sim\left[\left(\operatorname { c o s } ^ { 3 } ( \theta ) \operatorname { s i n } ( \theta ) \left(3 \chi_{x x x x}+2 \chi_{x x y y}-8 \chi_{x x z z}+\chi_{x y x y}+4 \chi_{z x z x}+\right.\right.\right. \\
& \left.\left.\left.\left(\chi_{x x x x}-2 \chi_{x x y y}-\chi_{x y x y}\right) \cos (\mathbf{4} \boldsymbol{\varphi})\right)+4\left(-2 \chi_{z z x x}+\chi_{z z z z}+\chi_{x z x z}\right) \sin ^{3}(\theta) \cos (\theta)\right)^{2}\right] \\
& \boldsymbol{I}^{P S}(\mathbf{2} \boldsymbol{\omega}) \sim\left[\left(-\chi_{x x x x}+2 \chi_{x x y y}+\chi_{x y x y}\right)^{2} \cos ^{4}(\theta) \sin ^{2}(\theta) \sin ^{2}(\mathbf{4} \boldsymbol{\varphi})\right] \\
& \boldsymbol{I}^{\boldsymbol{S P}}(\mathbf{2} \boldsymbol{\omega}) \sim\left[\left(-\chi_{z x z x} \cos (\theta) \sin (\theta)+\frac{1}{4} \cos (\theta)\left(\chi_{x x x x}-2 \chi_{x x y y}+3 \chi_{x y x y}+\left(-\chi_{x x x x}+\right.\right.\right.\right. \\
& \left.\left.\left.\left.2 \chi_{x x y y}+\chi_{x y x y}\right) \cos (\mathbf{4} \varphi)\right)\right)^{2} \sin ^{2}(\theta)\right] \\
& I^{S S}(\mathbf{2} \boldsymbol{\omega}) \sim\left[\left(-\chi_{x x x x}+2 \chi_{x x y y}+\chi_{x y x y}\right)^{2} \sin ^{2}(\theta) \sin ^{2}(\mathbf{4} \boldsymbol{\varphi})\right]
\end{aligned}
$$




$$
\begin{aligned}
& \text { I4, }\left\{\mathbf{4} / \mathbf{m m}^{\prime} \mathbf{m}^{\prime}(c)\right\}
\end{aligned}
$$

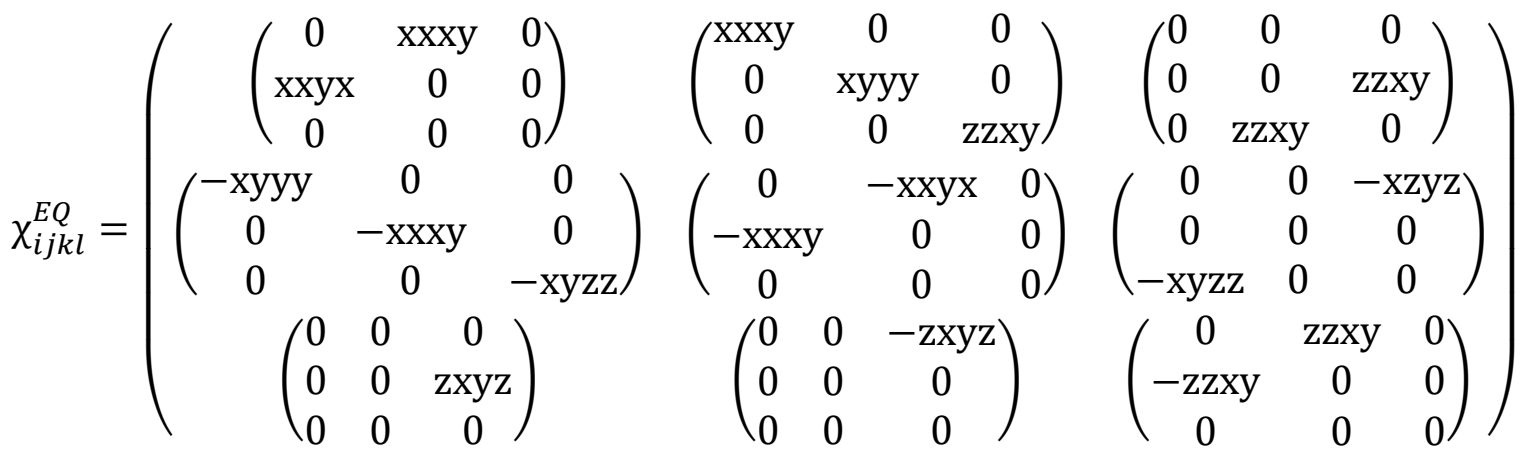

$$
\begin{aligned}
& \boldsymbol{I}^{P \boldsymbol{P}}(2 \boldsymbol{\omega}) \sim\left[\left(2 \chi_{\mathrm{xxxy}}+\chi_{\mathrm{xxyx}}-\chi_{\mathrm{xyyy}}\right)^{2} \cos ^{6}(\theta) \sin ^{2}(\theta) \sin ^{2}(4 \boldsymbol{\varphi})\right] \\
& \boldsymbol{I}^{P S}(\mathbf{2} \boldsymbol{\omega}) \sim\left[\operatorname { s i n } ^ { 2 } ( \theta ) \left(\operatorname { c o s } ^ { 2 } ( \theta ) \left(2 \chi_{\mathrm{xxxy}}+\chi_{\mathrm{xxyx}}-8 \chi_{\mathrm{xyzz}}+3 \chi_{\mathrm{xyyy}}\right.\right.\right. \\
& \left.\left.\left.\left.+\left(-2 \chi_{\mathrm{xxxy}}-\chi_{\mathrm{xxyx}}+\chi_{\mathrm{xyyy}}\right) \cos (4 \varphi)\right]\right)+4 \chi_{\mathrm{xzyz}} \sin ^{2}(\theta)\right)^{2}\right] \\
& \boldsymbol{I}^{\boldsymbol{S P}}(\mathbf{2} \boldsymbol{\omega}) \sim\left[\left(2 \chi_{\mathrm{xxxy}}+\chi_{\mathrm{xxyx}}-\chi_{\mathrm{xyyy}}\right)^{2} \cos ^{2}(\theta) \sin ^{2}(\theta) \sin ^{2}(\mathbf{4} \boldsymbol{\varphi})\right] \\
& \boldsymbol{I}^{S \boldsymbol{S}}(\mathbf{2} \boldsymbol{\omega}) \sim\left[\left(-2 \chi_{\mathrm{xxxy}}+3 \chi_{\mathrm{xxyx}}+\chi_{\mathrm{xyyy}}+\left(2 \chi_{\mathrm{xxxy}}+\chi_{\mathrm{xxyx}}-\chi_{\mathrm{xyyy}}\right) \cos (4 \varphi)\right)^{2} \sin ^{2}(\theta)\right]
\end{aligned}
$$




\section{J4, $\left\{\mathbf{4}^{\prime} / \mathbf{m m m}^{\prime}(\mathbf{c})\right\}$}

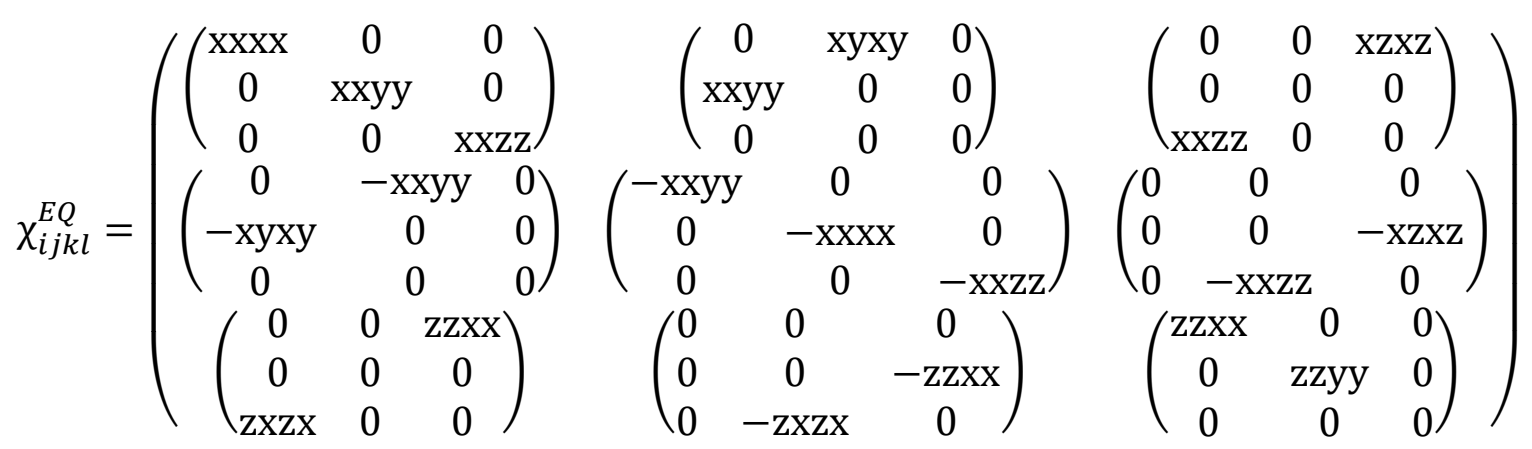

$$
\begin{gathered}
\boldsymbol{I}^{\boldsymbol{P P}}(\mathbf{2} \boldsymbol{\omega}) \sim\left[\cos (\theta) \sin ^{3}(\theta)\left(\chi_{z z x x}+\chi_{z z y y}-\left(2 \chi_{x z x z}-3 \chi_{z z x x}+\chi_{z z y y}\right) \cos ^{2}(\boldsymbol{\varphi})\right)\right. \\
\left.-\sin (\theta) \cos ^{3}(\theta)\left(\chi_{x x x x}-2 \chi_{x x z z}+\chi_{z x z x}\right) \cos ^{2}(\boldsymbol{\varphi})\right] \\
\boldsymbol{I}^{\boldsymbol{P S}}(\mathbf{2} \boldsymbol{\omega}) \sim\left[\left(\left(\chi_{x x x x}+2 \chi_{x x y y}-4 \chi_{x y x y}+\chi_{x y x y}\right) \sin (\theta) \cos ^{2}(\theta) \cos (\boldsymbol{\varphi}) \sin (\boldsymbol{\varphi})\right.\right. \\
\left.\left.+\chi_{x z x z} \sin ^{3}(\theta) \sin (2 \boldsymbol{\varphi})\right)^{2}\right] \\
\boldsymbol{I}^{\boldsymbol{S P}}(\mathbf{2} \boldsymbol{\omega}) \sim\left[\cos ^{2}(\theta) \sin ^{2}(\theta)\left(\left(-\chi_{x y x y}+\chi_{z x z x}\right) \cos (\mathbf{2} \boldsymbol{\varphi})\right)^{2}\right] \\
\boldsymbol{I}^{\boldsymbol{S S}}(\mathbf{2} \boldsymbol{\omega}) \sim\left[\left(\chi_{x x x x}-2 \chi_{x x y y}+\chi_{x y x y}\right)^{2} \sin ^{2}(\theta) \cos ^{2}(\boldsymbol{\varphi}) \sin ^{2}(\boldsymbol{\varphi})\right]
\end{gathered}
$$




\section{Comparison to GaAs}

To quantify the SHG intensity from $\mathrm{Sr}_{2} \mathrm{CuO}_{2} \mathrm{Cl}_{2}$, we perform measurements on $\mathrm{GaAs}$ - an inversion broken crystal often used as an SHG standard - under identical experimental conditions. As shown Figure S1, the RA-SHG intensity from $\mathrm{Sr}_{2} \mathrm{CuO}_{2} \mathrm{Cl}_{2}$ is approximately $10^{4}$ times smaller than that of GaAs (001).

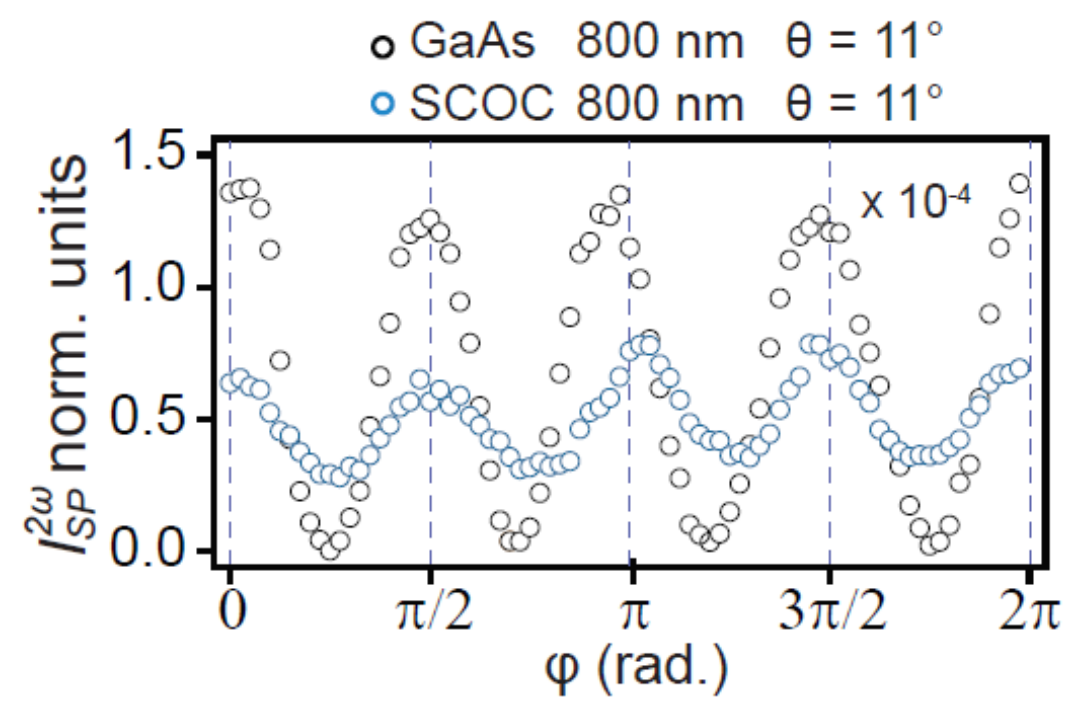

Figure S1: RA-SHG data from $\mathrm{Sr}_{2} \mathrm{CuO}_{2} \mathrm{Cl}_{2}$ and GaAs (scaled by $10^{-4}$ ) measured at $T=300 \mathrm{~K}$ in the SP channel under identical experimental conditions. 


\section{Simulated RA-SHG patterns using AFM point group}

Figure S2 shows simulated RA-SHG patterns due to interference between an MD or EQ process from the magnetic point group $\mathrm{mmm} 1^{\prime}$ and an EQ process from the crystallographic point group $4 / \mathrm{mmm}$. As expected, the resulting RA-SHG patterns break $\mathrm{C}_{4}$ as well as the $a c$ and $b c$ mirror planes but preserve the $x z$ and $y z$ mirror planes, making them incompatible with our data.
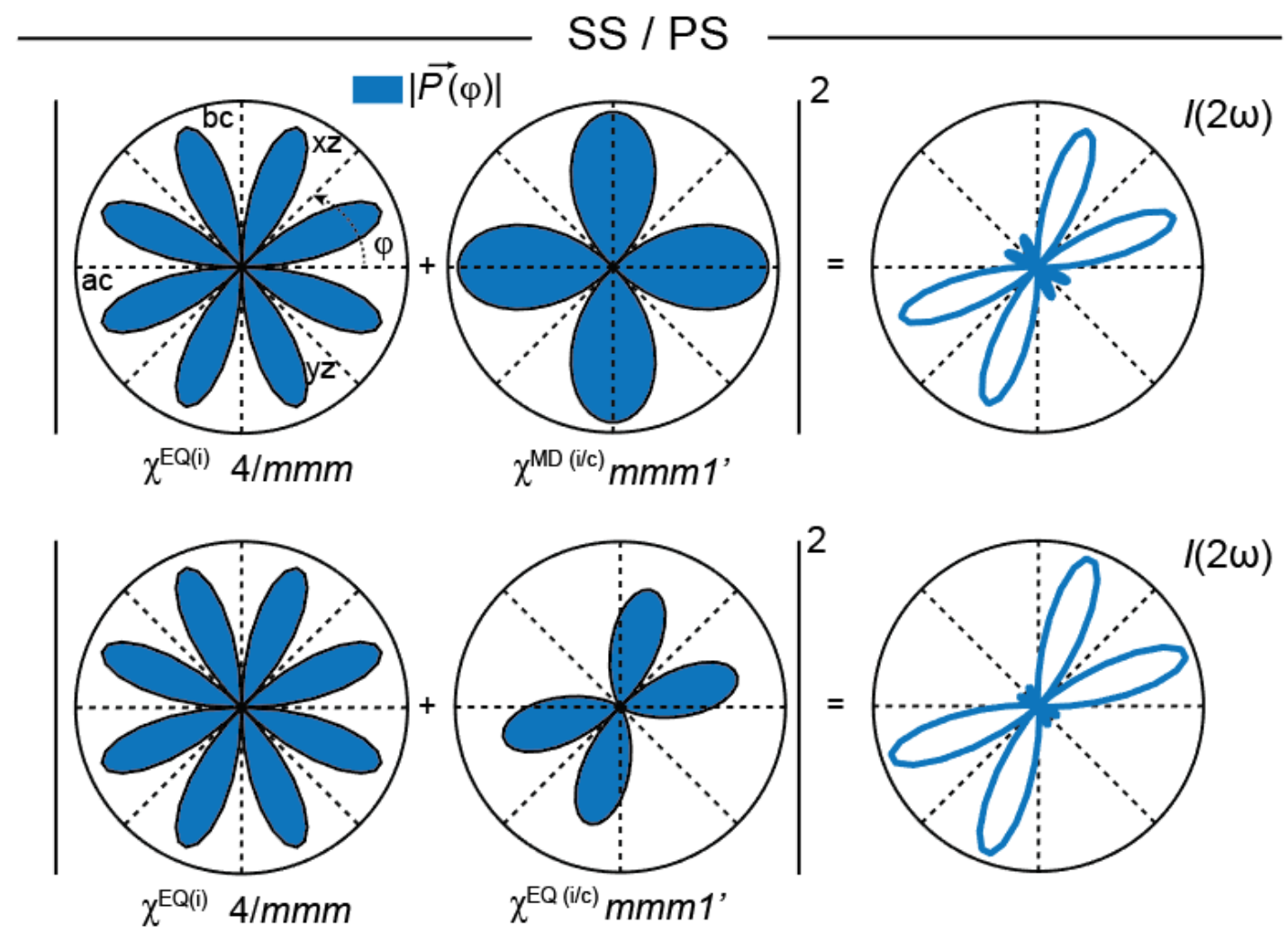

Figure S2: Simulated RA-SHG patterns assuming constructive interference between EQ SHG from 4/mmm and (top) MD or (bottom) EQ SHG from $m m m 1^{\prime}$. An arbitrary set of susceptibility tensor element values were used. These simulations are representative of both the PS and SS channels. 


\section{Description of possible $A_{2 g}$ order parameters}

As discussed in the main text, the order parameter associated with the $4 / \mathrm{mm}^{\prime} \mathrm{m}^{\prime}$ magnetic point group has $A_{2 \mathrm{~g}}$ symmetry. The simplest state that realizes this symmetry is a ferromagnet with a moment along $c$. But, as mentioned in the main text, canting has been ruled out by XMCD and is also inconsistent with $I 4 / \mathrm{mmm}$, which does not allow for a DM interaction. On the other hand, magneto-chiral phases with this symmetry do exist, and are equivalent to an orbital ferromagnet with moment along $c$ [Fig. S3(a)]. We note that below $T_{\mathrm{N}}$, the actual magnetic point group of $\mathrm{Sr}_{2} \mathrm{CuO}_{2} \mathrm{Cl}_{2}$ would be $m m^{\prime} m^{\prime}$, which is the intersection of $m m m 1^{\prime}$ with $4 / \mathrm{mm}^{\prime} m^{\prime}$.

In general, any axial harmonic with odd $L$ and $M=0$ has $A_{2 g}$ symmetry. An example is the $(3,0)$ component of a magnetic octupole, which is illustrated in Figure S3(b). While we do not make any claims about its microscopic origin, we note that a ferroic ordering of $(3,0)$ magnetic octupoles would be difficult to detect by more conventional methods like neutron or x-ray diffraction.

(a)

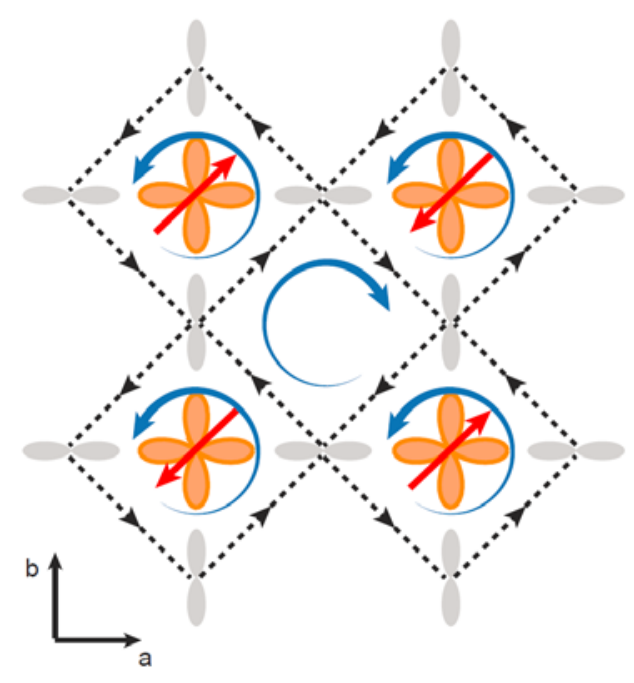

(b)

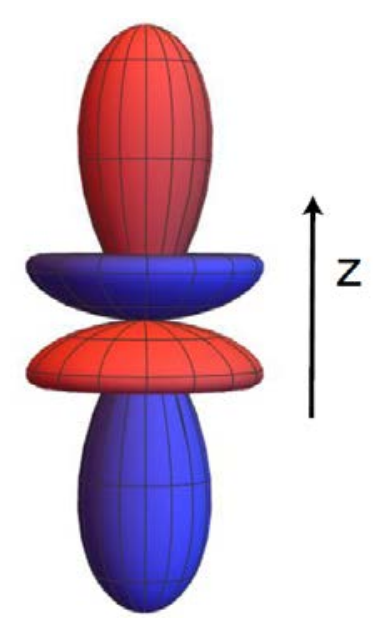

Figure S3: (a) Magneto-chiral loop current phase (dashed black arrows) superimposed on the AFM state (red arrows). The blue arrows schematically show the direction of orbital currents. Orange (gray) lobes denote the copper (oxygen) orbitals. (b) Schematic of the $(3,0)$ component of a magnetic octupole. Red and blue encode opposite phase. 


\section{Temperature dependence of normal incidence intensity}

Figure S4 shows the temperature dependence of the normal incidence SHG response of $\mathrm{Sr}_{2} \mathrm{CuO}_{2} \mathrm{Cl}_{2}$ in both parallel (X-X) and perpendicular (X-Y) polarization channels. No discernible SHG signal is observed at any temperature.

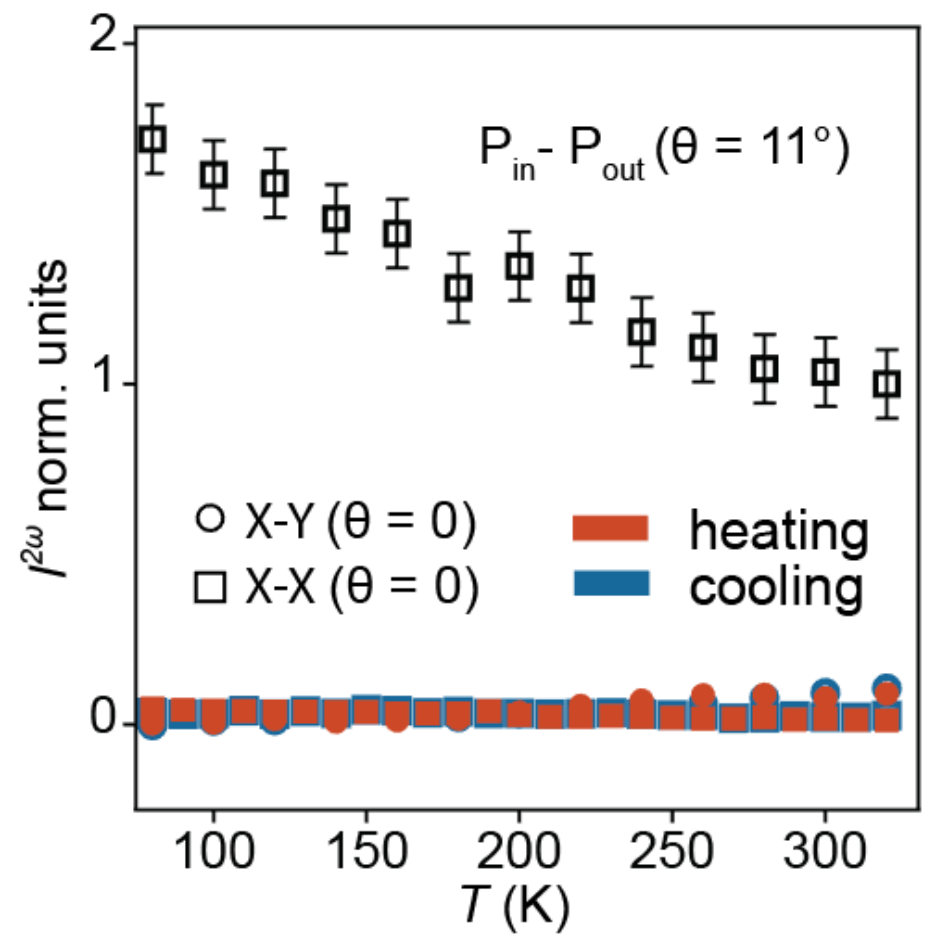

Figure S4: Temperature dependence of normal incidence SHG intensity for X-X and X-Y polarization channels measured using $800 \mathrm{~nm}$ light. The oblique incidence $\left(\theta=11^{\circ}\right)$ SHG intensity measured in the PP channel with 800 nm light at $\varphi=\pi / 4$ is shown for comparison. All data are normalized to the room temperature value of the PP curve. 


\section{Temperature dependence of intensity in PS and PP channels}

Figure S5 shows the SHG intensity versus temperature in the PS and PP channels. It confirms that the feature at $T_{\mathrm{N}}$ is only clearly observable in the S-output channels.
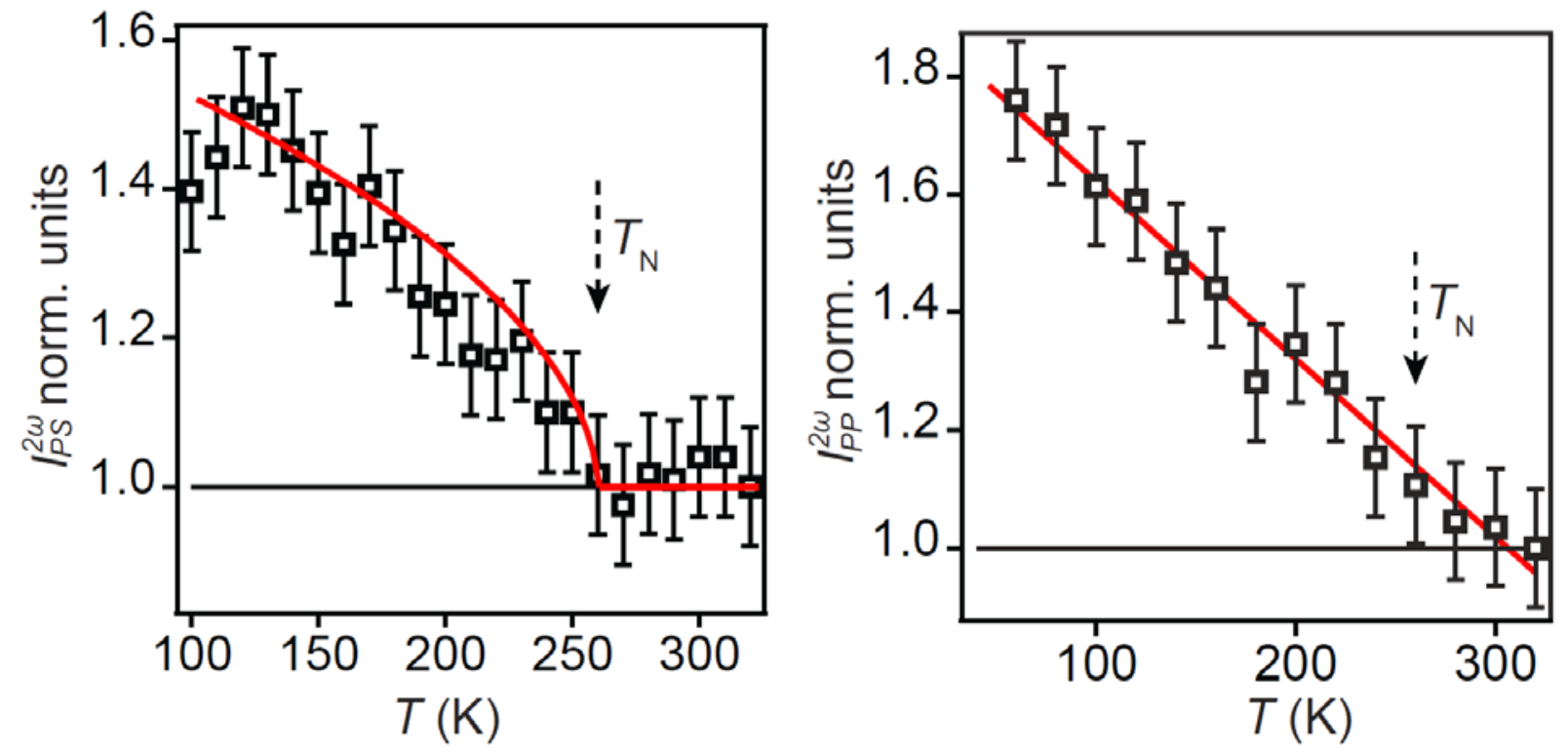

Figure S5: SHG intensity measured in the PS $(\varphi=3 \pi / 8)$ and PP $(\varphi=\pi / 4)$ channels at normalized to their $T=320 \mathrm{~K}$ values. Red lines are guides to the eye. 


\section{Temperature and $\varphi$-dependence of intensity in SP and PP channels}

To confirm that the lack of any feature at $T_{\mathrm{N}}$ in the P-output channels is true at any $\varphi$, we collected full RA-SHG patterns as a function of temperature. As shown in Figure S6, no abrupt change of intensity or symmetry is observed near $T_{\mathrm{N}}$ at all $\varphi$.

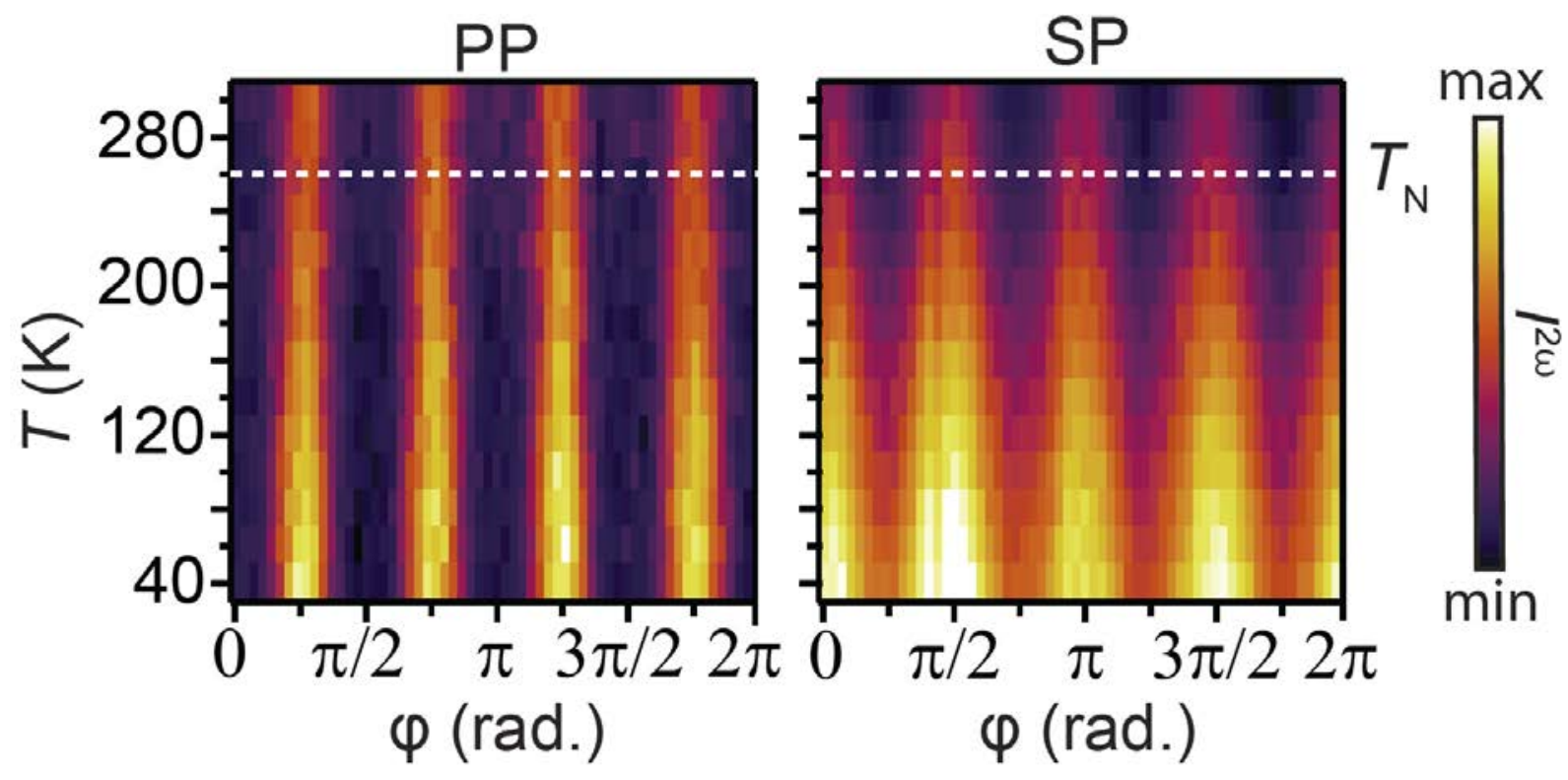

Figure S6: Temperature dependence of RA-SHG patterns in the P-output channels. 


\section{Effects of non-uniform lattice contraction}

A non-uniform contraction of the $a$ and $c$ lattice constants of $\mathrm{Sr}_{2} \mathrm{CuO}_{2} \mathrm{Cl}_{2}$ has been reported by neutron diffraction ${ }^{3}$ and is shown in Figure S7(a). This change in lattice constants will change the relative magnitudes of the $4 / \mathrm{mmm}$ allowed crystallographic EQ SHG tensor elements. The significantly larger fractional contraction along the $c$-axis should lead to a stronger temperature dependence of the $\chi_{i j k l}^{E Q(i)}$ tensor elements with $i, j$ or $l=z$, which only appear in the P-output channels. As a check, we fit the $T=320 \mathrm{~K}$ and $T=20 \mathrm{~K}$ data collected in the P-output channels to the function $(a+b \cos 4 \varphi)^{2}$. As shown in section $1, b=\left(\chi_{x x x x}-2 \chi_{x x y y}-\chi_{x y x y}\right)$ while the aforementioned $z$ containing $\chi_{i j k l}^{E Q(i)}$ elements are all included in $a$. We first fit the $T=320 \mathrm{~K}$ data and obtained the following fit values. PP: ( $a=0.71, b=-0.24)$, SP: $(a=0.95, b=0.18)$. We then fit the $T=20 \mathrm{~K}$ data by keeping the $b$ 's fixed to their high temperature values and letting only the $a$ 's be free parameters. We obtained PP ( $a=0.89, b=-0.24)$, SP $(a=1.60, b=0.18)$. As shown in Figure S7(b), the temperature dependence of the RA-SHG patterns is very well captured by only changing the $a$ terms. Allowing $a$ to be complex does not change the conclusions.

(a)

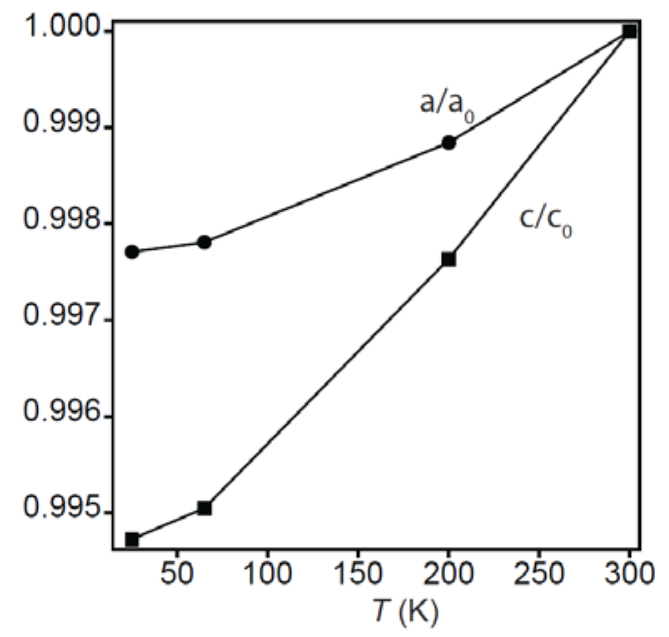

(b)
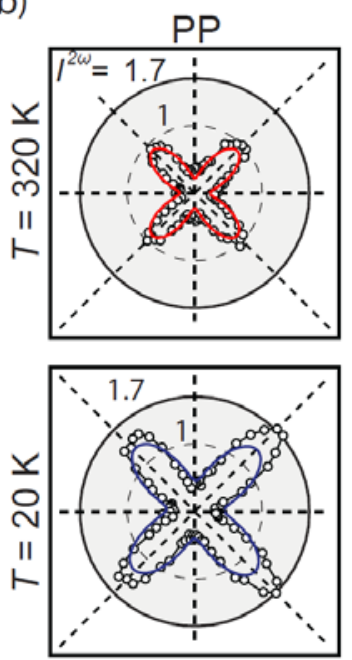
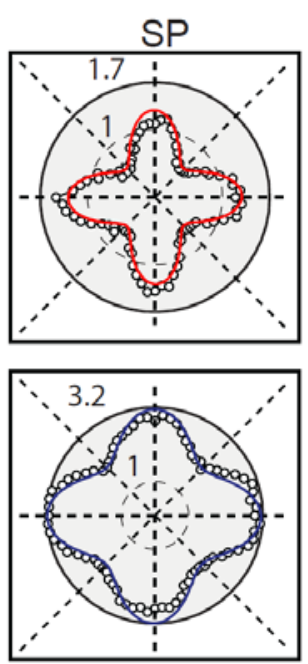

Figure S7: (a) Temperature dependence of the lattice parameters of $\mathrm{Sr}_{2} \mathrm{CuO}_{2} \mathrm{Cl}_{2}$ reported by neutron diffraction ${ }^{3}$. (b) Fits (red/blue lines) to the RA-SHG data (circles/black lines) in the P-output channels. Fits to the $T=20 \mathrm{~K}$ data were obtained by allowing only $a$ to vary and leaving $b$ fixed to its $T=320 \mathrm{~K}$ value. 


\section{Laser fluence and average power considerations}

As discussed in the Methods section, a minimum laser fluence of around $3 \mathrm{~mJ} / \mathrm{cm}^{2}$ at $1.5 \mathrm{eV}$ photon energy was necessary to acquire RA-SHG patterns (especially in the SS channel) of reasonable quality. Based on prior time-resolved optical reflectivity measurements on $\mathrm{Sr}_{2} \mathrm{CuO}_{2} \mathrm{Cl}_{2}{ }^{4}$, no evidence of optical saturation is reported at this fluence level even at $3 \mathrm{eV}$ photon energy, where the absorption is much higher than at $1.5 \mathrm{eV}$.

We estimate an upper limit to the steady state temperature increase of the sample $(\Delta T)$ using the formula for the average heating of a Gaussian beam $\Delta T=\sqrt{\frac{\ln 2}{\pi}}\left(\frac{P}{\kappa l}\right)$. Here $P$ is the average laser power, $\kappa$ is the thermal conductivity and $l$ is the FWHM of our Gaussian beam spot. To achieve a fluence of $3 \mathrm{~mJ} / \mathrm{cm}^{2}$ we use $P=3 \mathrm{~mW}$ and $l=40 \mu \mathrm{m}$. Plugging in the reported in-plane value for the thermal conductivity ${ }^{5}$ at $260 \mathrm{~K}(\kappa=10 \mathrm{~W} / \mathrm{K} . \mathrm{m})$ we obtain $\Delta T=3.5 \mathrm{~K}$. This is consistent with the negligible difference between the value of $T_{\mathrm{N}}$ observed in our SHG experiment and that reported in the literature. 


\section{Effects of thermal cycling}

In attempt to stabilize a region with a time-reversed order parameter $(-\Phi)$, we collected RA-SHG data in the SS channel from the same spots on $\mathrm{Sr}_{2} \mathrm{CuO}_{2} \mathrm{Cl}_{2}$ under repeated thermal cycles across $T_{\mathrm{N}}$. As shown in Figure S8, upon thermal cycling, the relative magnitude of adjacent lobes at low temperature does not change. This means that the sign of $\Phi$ does not flip.
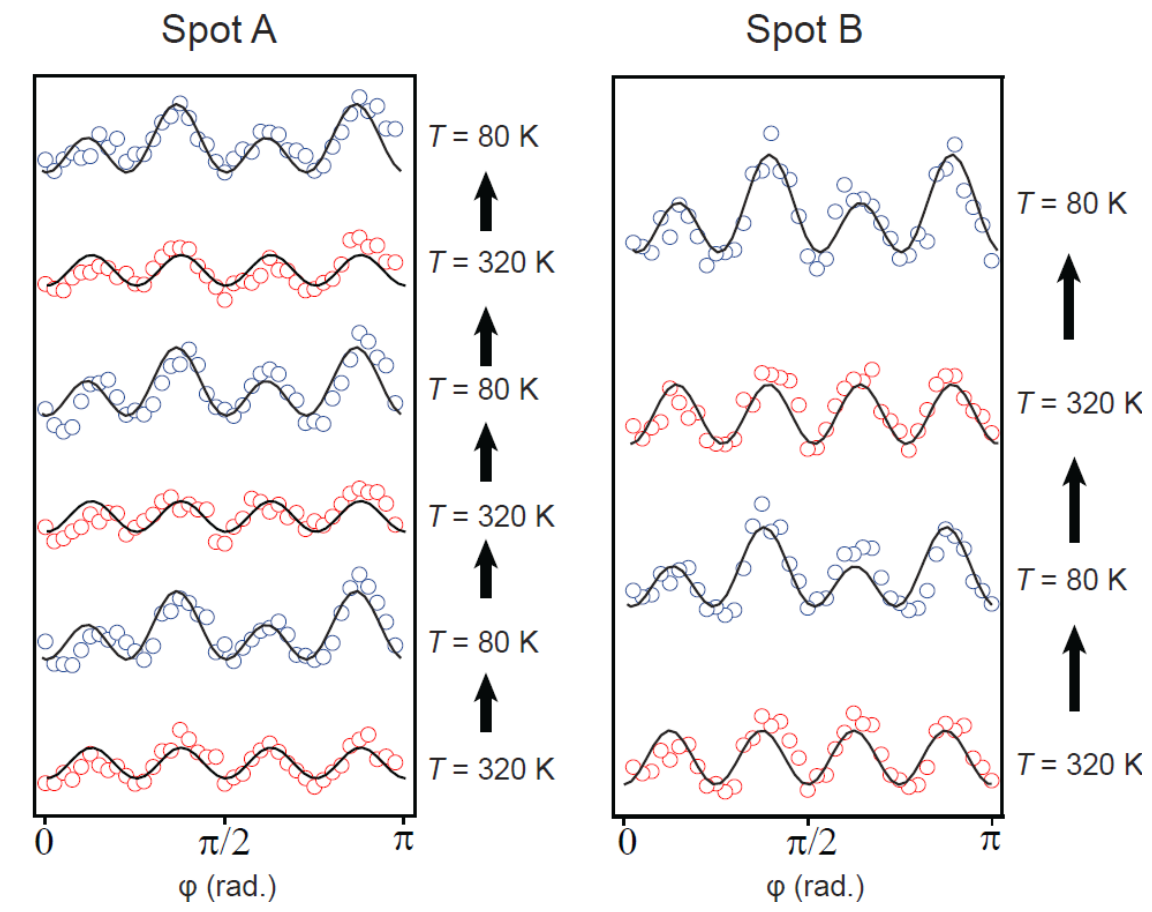

Figure S8: RA-SHG data in the SS channel measured from the same spot on $\mathrm{Sr}_{2} \mathrm{CuO}_{2} \mathrm{Cl}_{2}$ upon successive thermal cycles at two different locations. Curves are vertically offset for clarity. Lines are guides to the eye. 


\section{Discussion of the effects of crystal imperfections}

We discuss several types of possible imperfections in the crystallographic and magnetic structures of $\mathrm{Sr}_{2} \mathrm{CuO}_{2} \mathrm{Cl}_{2}$ and their possible influence on the SHG response.

(1) While the average structure of $\mathrm{Sr}_{2} \mathrm{CuO}_{2} \mathrm{Cl}_{2}$ is known to have a 4/mmm point group, it is possible that the local structure has lower symmetry. This can lead to a finite local spin canting even though it is forbidden in the average structure. Below we present several reasons why we can exclude local distortions, either dynamic or static, as the source of our SHG signal.

Consider dynamical fluctuations that generate a finite instantaneous spin canting, which timeaverages to zero. Since our experiments average over many optical pulses, we are measuring a time-average over all instantaneous spin configurations, yielding no net symmetry breaking.

Next consider local static distortions that cause local static spin canting (either up or down), which averages to zero over space. If the characteristic structural modulation wavelength is much shorter than our optical wavelength $(800 \mathrm{~nm})$, then the SHG contributions from up and down regions add coherently, canceling out any spin-dependent contribution to SHG. If the modulation wavelength is longer than our optical wavelength, then the SHG contributions from up and down regions add incoherently, averaging away any symmetry reduction in the RA patterns.

(2) It is likely there are edge and/or screw dislocations in $\mathrm{Sr}_{2} \mathrm{CuO}_{2} \mathrm{Cl}_{2}$ as a result of the crystal growth. This can lead to local crystallographic and spin structures that are different than the bulk. Below we outline reasons why such effects do not explain the SHG data. First, the new SHG contribution below $T_{\mathrm{N}}$ is the same order of magnitude as the coherent EQ contribution above $T_{\mathrm{N}}$. It is difficult to justify how a sparse density of dislocations and/or magnetic texture defects can generate such a large signal. Second, SHG from random scattering centers, either structural or magnetic, would be diffuse (hyper-Rayleigh) in nature ${ }^{6}$. But our SHG signal is highly specular, with no evidence of an increasing diffuse contribution below $T_{\mathrm{N}}$. Third, dislocations and their induced magnetic texture defects would be expected to break the tetragonal symmetry of the average structure. This is hard to reconcile with the relatively high $4 / \mathrm{mm}^{\prime} \mathrm{m}^{\prime}$ symmetry observed. 


\section{Comparison to prior SHG work on YBCO}

Previous RA-SHG measurements on doped $\mathrm{YBa}_{2} \mathrm{Cu}_{3} \mathrm{O}_{\mathrm{y}}$ revealed a loss of vertical mirror planes $^{7}$, despite diffraction based measurements showing a crystallographic structure (point group $\mathrm{mmm}$ ) that preserves them. This RA signal is already present at room temperature and is enhanced below the pseudogap temperature $T^{*}$. To explain this data, it was proposed that there already exists a subtle monoclinic structural distortion at room temperature, and that a further inversion-breaking transition occurs at $T^{*}$. The low temperature RA data could be fit to the coherent sum of a crystallographic $i$-type $C_{2}$-symmetric EQ term (point group $2 / m$ ) and a domain-averaged $c$-type $C_{1}$-symmetric ED term (point group $2^{\prime} / m$ or $m 1^{\prime}$ ).

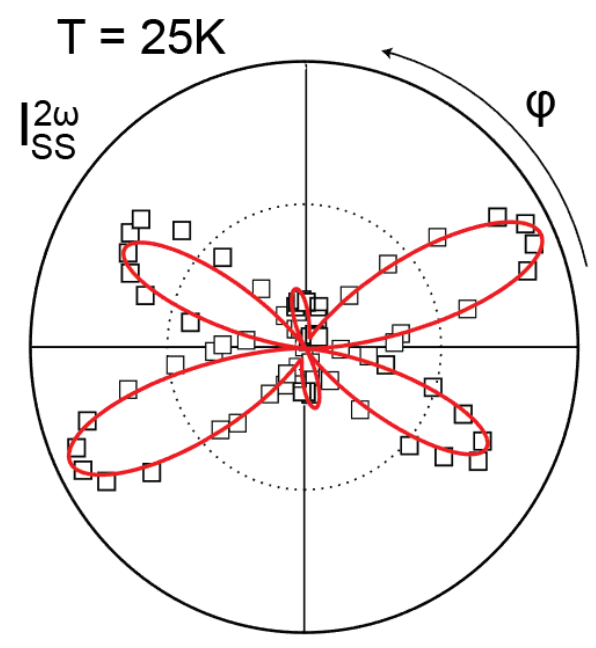

Figure S9: RA data from $\mathrm{YBa}_{2} \mathrm{Cu}_{3} \mathrm{O}_{6.92}$ at $T=25 \mathrm{~K}$ in the $\mathrm{SS}$ channel reproduced from Ref.[6]. The red curve is a fit the superposition of an $i$-type EQ term $(\mathrm{mmm})$ and a $c$-type MD term $\left(\mathrm{mm}^{\prime} \mathrm{m}^{\prime}\right)$ from a single magnetic domain.

Here we show that the RA patterns from $\mathrm{YBa}_{2} \mathrm{Cu}_{3} \mathrm{O}_{\mathrm{y}}$ can also be explained by the presence of an inversion-preserving order parameter $\Phi$ of the type we report in $\mathrm{Sr}_{2} \mathrm{CuO}_{2} \mathrm{Cl}_{2}$. In direct analogy to $\mathrm{Sr}_{2} \mathrm{CuO}_{2} \mathrm{Cl}_{2}$, we fit the RA data from $\mathrm{YBa}_{2} \mathrm{Cu}_{3} \mathrm{O}_{\mathrm{y}}$ to the sum of a crystallographic $i$-type EQ term respecting the reported $\mathrm{mmm}$ point group, and a $c$-type MD term with a $\mathrm{mm}^{\prime} \mathrm{m}^{\prime}$ point group from a single domain. In the SS channel, the functional form of the RA pattern is given by

$$
I_{S S}^{2 \omega} \propto\left|\left(a \cos ^{3} \varphi \sin \varphi+b \cos \varphi \sin ^{3} \varphi\right)+\left(c \cos ^{2} \varphi+d \sin ^{2} \varphi\right)\right|^{2}
$$

where $a=\chi_{x y x y}^{E Q(i)}+\chi_{y x x y}^{E Q(i)}+\chi_{y y x x}^{E Q(i)}-\chi_{y y y y}^{E Q(i)}, b=\chi_{x x x x}^{E Q(i)}-2 \chi_{x x y y}^{E Q(i)}-\chi_{y x y x}^{E Q(i)}, c=\chi_{y y z}^{M D(c)}$ and $d=\chi_{x x z}^{M D(c)}$. As shown in Fig. S9, this also fits the $\mathrm{YBa}_{2} \mathrm{Cu}_{3} \mathrm{O}_{\mathrm{y}}$ data extremely well. 


\section{Supplementary references}

1. Varma, C. M. Pseudogap and Fermi arcs in underdoped cuprates. Physical Review B 99, 224516 (2019).

2. Birss, R. R. Symmetry and magnetism. (John Wiley \& Sons, 1964).

3. Miller, L. L. et al. Synthesis, structure, and properties of $\mathrm{Sr}_{2} \mathrm{CuO}_{2} \mathrm{Cl}_{2}$. Phys. Rev. B 41, 19211925 (1990).

4. Sahota, D. G. et al. Many-body recombination in photoexcited insulating cuprates. Phys. Rev. Research 1, 033214 (2019).

5. Hofmann, M. et al. Evidence for a large magnetic heat current in insulating layered cuprates. Phys. Rev. B 67, 184502 (2003).

6. Jeong, J.-W., Shin, S.-C., Lyubchanskii, I. L. \& Varyukhin, V. N. Strain-induced three-photon effects. Phys. Rev. B 62, 13455-13463 (2000).

7. Zhao, L. et al. A global inversion-symmetry-broken phase inside the pseudogap region of $\mathrm{YBa}_{2} \mathrm{Cu}_{3} \mathrm{O}_{y}$. Nature Physics 13, 250-254 (2017). 